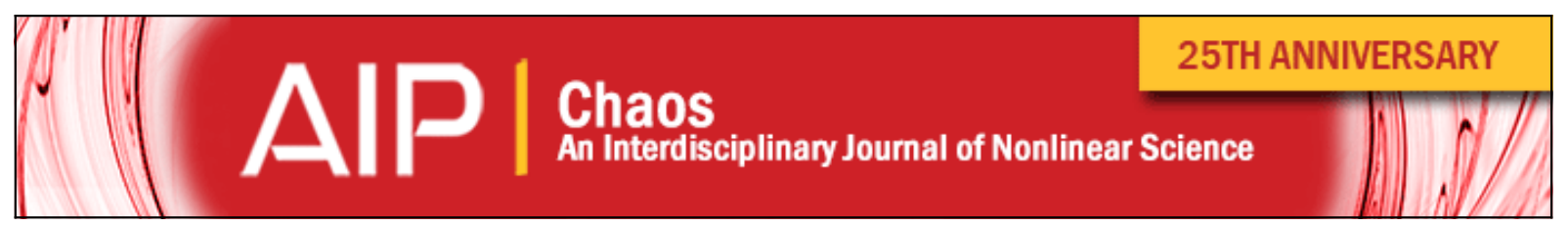

\title{
Transient and chaotic low-energy transfers in a system with bistable nonlinearity
}

F. Romeo, L. I. Manevitch, L. A. Bergman, and A. Vakakis

Citation: Chaos: An Interdisciplinary Journal of Nonlinear Science 25, 053109 (2015); doi: 10.1063/1.4921193

View online: http://dx.doi.org/10.1063/1.4921193

View Table of Contents: http://scitation.aip.org/content/aip/journal/chaos/25/5?ver=pdfcov

Published by the AIP Publishing

\section{Articles you may be interested in}

Synchronization, non-linear dynamics and low-frequency fluctuations: Analogy between spontaneous brain activity and networked single-transistor chaotic oscillators

Chaos 25, 033107 (2015); 10.1063/1.4914938

On the modeling and nonlinear dynamics of autonomous Silva-Young type chaotic oscillators with flat power spectrum

Chaos 24, 043134 (2014); 10.1063/1.4903313

Global synchronization in lattices of coupled chaotic systems

Chaos 17, 033111 (2007); 10.1063/1.2754668

A new scheme to generalized (lag, anticipated, and complete) synchronization in chaotic and hyperchaotic systems

Chaos 15, 013101 (2005); 10.1063/1.1827431

Control of noisy chaotic motion in a system with nonlinear excitation and restoring forces

Chaos 7, 290 (1997); 10.1063/1.166228

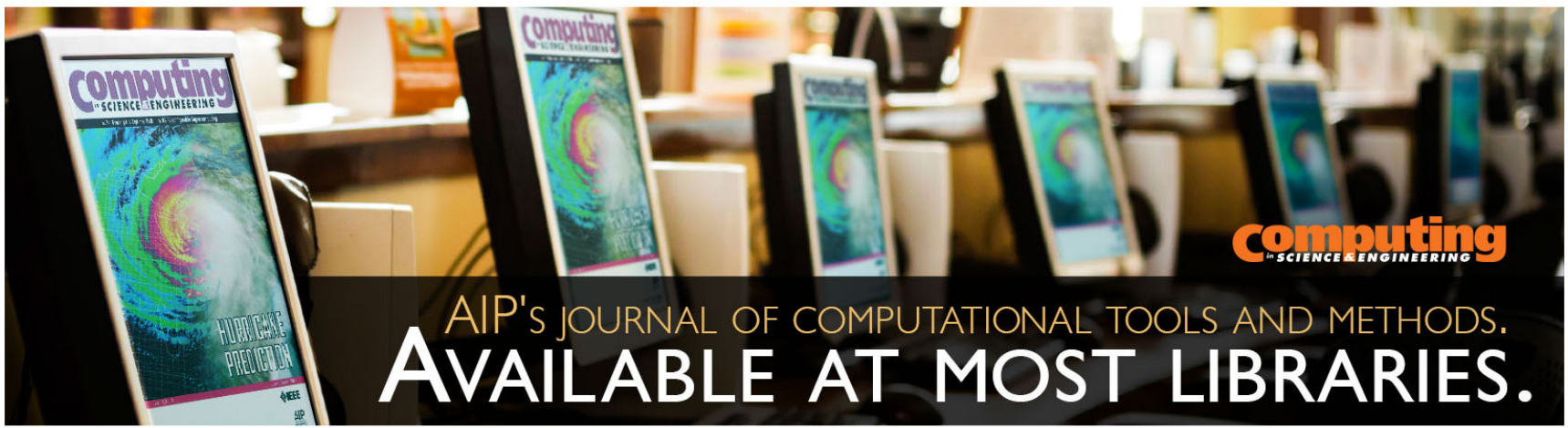




\title{
Transient and chaotic low-energy transfers in a system with bistable nonlinearity
}

\author{
F. Romeo, ${ }^{1, a)}$ L. I. Manevitch, ${ }^{2}$ L. A. Bergman, ${ }^{3}$ and A. Vakakis ${ }^{3}$ \\ ${ }^{1}$ Department of Structural and Geotechnical Engineering, SAPIENZA University of Rome, Rome, Italy \\ ${ }^{2}$ Institute of Chemical Physics, RAS, Moscow, Russia \\ ${ }^{3}$ College of Engineering, University of Illinois at Urbana-Champaign, Champaign, Illinois 61820, USA
}

(Received 15 January 2015; accepted 4 May 2015; published online 18 May 2015)

\begin{abstract}
The low-energy dynamics of a two-dof system composed of a grounded linear oscillator coupled to a lightweight mass by means of a spring with both cubic nonlinear and negative linear components is investigated. The mechanisms leading to intense energy exchanges between the linear oscillator, excited by a low-energy impulse, and the nonlinear attachment are addressed. For lightly damped systems, it is shown that two main mechanisms arise: Aperiodic alternating in-well and cross-well oscillations of the nonlinear attachment, and secondary nonlinear beats occurring once the dynamics evolves solely in-well. The description of the former dissipative phenomenon is provided in a two-dimensional projection of the phase space, where transitions between in-well and cross-well oscillations are associated with sequences of crossings across a pseudo-separatrix. Whereas the second mechanism is described in terms of secondary limiting phase trajectories of the nonlinear attachment under certain resonance conditions. The analytical treatment of the two aformentioned low-energy transfer mechanisms relies on the reduction of the nonlinear dynamics and consequent analysis of the reduced dynamics by asymptotic techniques. Direct numerical simulations fully validate our analytical predictions. (C) 2015 AIP Publishing LLC.
\end{abstract}

[http://dx.doi.org/10.1063/1.4921193]

A system consisting of a grounded linear oscillator (LO) with a strongly nonlinear ungrounded attachment possessing a stiffness with combined negative linear and cubic nonlinear terms is considered. This type of nonlinear attachment is capable of absorbing passively the energy initially induced in the linear oscillator over a broad range of input energy. Accordingly, this configuration overcomes the limitations of the pure cubic nonlinear attachment for which a well-defined threshold of input energy exists below which no significant energy absorption and dissipation can be achieved. The mechanisms leading to intense energy exchanges between the linear oscillator, excited by a low-energy impulse, and the nonlinear attachment are addressed. The proposed approaches describe and predict the beneficial dynamic phenomena governing the targeted energy transfer from the linear oscillator to the nonlinear attachment, thus providing valuable tools to design efficient passive energy absorption devices for low amplitude ambient vibrations.

\section{INTRODUCTION}

A system consisting of a grounded linear oscillator with a strongly nonlinear ungrounded attachment possessing a stiffness with combined negative linear and cubic nonlinear terms is considered (Figure 1). As recently shown in Refs. 1 and 2, this type of nonlinear attachment is capable of absorbing passively the energy initially induced in the linear

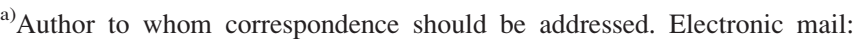
francesco.romeo@uniroma1.it.
}

oscillator over a broad range of input energy. Accordingly, this configuration overcomes the limitations of the pure cubic nonlinear attachment for which a well-defined threshold of input energy exists below which no significant energy absorption and dissipation can be achieved. ${ }^{3}$

Whereas many works have considered the nonlinear dynamics of systems possessing bistable elements ${ }^{4-10}$ or negative stifffness, ${ }^{1-16}$ the combined effects of both such types of stiffness characteristics have only recently been explored. ${ }^{1,2}$ On the other hand, the capacity to build nonlinear springs with complex stiffness characteristics (e.g., negative and nonlinear force-displacement laws) has been demonstrated in Ref. 17, paving the way of utilizing such complex nonlinear springs in practical designs of mechanical components.

In Refs. 1 and 2, an impulsively forced linear oscillator coupled to a (non-directly forced) lightweight attachment by means of a complex spring combining negative linear and hardening cubic stiffness characteristics was considered and

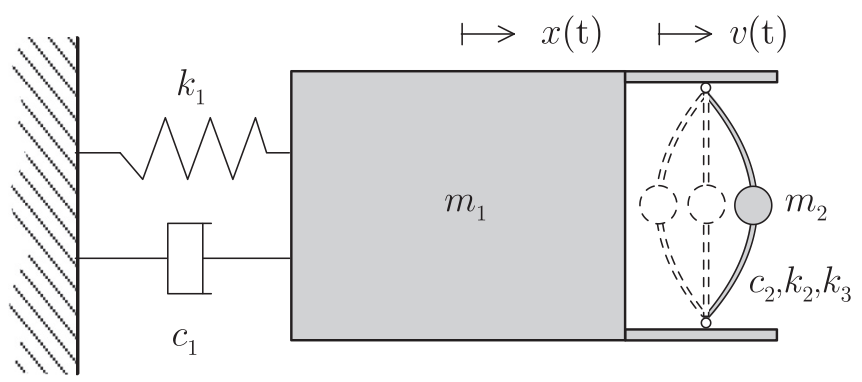

FIG. 1. The model consisting of a linear oscillator of mass $m_{1}$ coupled to a lightweight attachment $m_{2}$ through a buckled beam, with negative linear $\left(k_{2}\right)$ and cubic nonlinear $\left(k_{3}\right)$ stiffness coefficients. 
nonlinear targeted energy transfers from the linear oscillator to the nonlinear attachment were analytically and numerically studied. According to the intensity of the input energy applied to the LO, different mechanisms explain the passive energy absorption by the nonlinear attachment (termed from now on as nonlinear energy sink-NES). In particular, as shown in Ref. 2, for high input energy, similar to the pure cubic NES configuration, the transient 1:1 resonant capture is the leading mechanism for energy transfer from the LO to the NES, and high amplitude periodic oscillations of the NES occur; whereas as the input energy decreases to intermediate values, nonlinear beats provide the most efficient energy transfer mechanism in that case. For both high and intermediate energy regimes, however, the nonlinearity generated by the pure cubic stiffness of the NES dominates over the negative stiffness effects. This changes, however, at low energy inputs. Indeed, at low input excitations, the bistability of the NES triggers interesting transient dynamics evolving around the two potential wells of the dynamics of the system, where aperiodic cross-well oscillations represent the most beneficial energy transfer mechanism. Furthermore, in-well nonlinear beats can be also observed at very low energy levels during the later stages of the transient dynamics.

The bistable NES considered herein possesses two nontrivial stable equilibria and one trivial unstable equilibrium; moreover, its phase-plane is divided in two regions and its transient dynamics is characterised by in-well as well as cross-well oscillations, depending on which region is visited by the orbits of the system. We will show in this work that when a low energy input is applied to the LO, these bistability features affect significantly the transient dynamics of the entire coupled system.

In particular, we aim to describe in detail the nonlinear targeted energy transfer mechanisms occurring at low energy in this system and provide predictive design capacity which will be based on the understanding and analysis of the critical nonlinear dynamics that govern these low-energy transfers. The present work is organised as follows. In Sec. II, the main energy transfer mechanisms and their performance in terms of rate of energy dissipated by the NES are summarized and discussed. In Sec. III, the influence of the negative stiffness on broadening the NES response spectrum for low input energy is highlighted and the pseudo-separatrix concept is introduced in anticipation of the order reduction carried out in Sec. IV. Next, in Sec. IV, the reduction of the system dynamics to a single degree of freedom (SDOF) reduced-order system is introduced aiming to analyze the low-energy chaotic transitions by means of Melnikov analysis and Lyapunov characteristic exponents. In Sec. V, the in-well nonlinear beats occurring at very low energy levels are described as secondary Limiting Phase Trajectories (LPTs). Finally, some concluding remarks pointing toward future experimental investigations are reported in the last section.

\section{MAIN ENERGY TRANSFER MECHANISMS}

The system under investigation consists of a linear primary oscillator of mass $m_{1}$ (the LO) and viscous damping coefficient $c_{1}$ that is coupled to a lightweight mass $m_{2}$ (the nonlinear attachment, bistable NES), with viscous damping coefficient $c_{2}$, by means of negative linear $\left(k_{2}\right)$ and cubic nonlinear $\left(k_{3}\right)$ springs in parallel. The mechanical design corresponding to the considered bistable NES model can be realized in different ways. The simplest one is sketched in Figure 1, where a buckled beam with a midspan point mass is rigidly coupled to the primary linear oscillator. The level of pre-compression in the added beam, the straight configuration of which is unstable, governs the relationship between positive cubic and negative linear stiffness components. The equations of motion of this two-DOF system are given by

$$
\begin{gathered}
m_{1} \ddot{x}+k_{1} x+c_{1} \dot{x}+k_{2}(x-v)+c_{2}(\dot{x}-\dot{v})+k_{3}(x-v)^{3}=0, \\
m_{2} \ddot{v}+k_{2}(v-x)+c_{2}(\dot{v}-\dot{x})+k_{3}(v-x)^{3}=0 .
\end{gathered}
$$

Being interested in the transient dynamics, the system (1) is studied under the following initial conditions at $t=0$, $x=v=0, \dot{x}=X>0, \dot{v}=0$. By setting

$$
\begin{gathered}
\varepsilon=\frac{m_{2}}{m_{1}} ; \quad \omega_{0}^{2}=\frac{k_{1}}{m_{1}} ; \quad C_{0}=\frac{k_{2}}{m_{1}} ; \quad C=\frac{k_{3}}{m_{1}} ; \\
\lambda_{1}=\frac{c_{1}}{m_{1}} ; \quad \lambda_{2}=\frac{c_{2}}{m_{1}},
\end{gathered}
$$

and introducing the change of variables

$$
v=z+d \quad d=\sqrt{\left|\frac{C_{0}}{C}\right|},
$$

the equations of motion (2) can be rewritten in normalized form as

$$
\begin{aligned}
\ddot{x} & +\omega_{0}^{2} x+\left(C_{0}+3 C d^{2}\right)(x-z)+C\left[(x-z)^{3}-3 d(x-z)^{2}\right] \\
& +\lambda_{1} x+\lambda_{2}(\dot{x}-\dot{z})=0, \quad \varepsilon \ddot{z}+\left(C_{0}+3 C d^{2}\right)(z-x) \\
& +C\left[(z-x)^{3}+3 d(z-x)^{2}\right]+\lambda_{2}(\dot{z}-\dot{x})=0 .
\end{aligned}
$$

As shown in Refs. 1 and 2, three energy regions with different main energy transfer mechanisms exist based on the frequency-energy plot of the underlying Hamiltonian system. The main backbone branches (composed of periodic solutions under condition of 1:1 resonance of the underlying system without damping or forcing) showed that the negative stiffness affects the width of the energy interval corresponding to intensive energy transfer from the LO to the NES. In particular, the latter favorable regime is due to nonlinear beats and arises within the region of existence of out-of-phase unstable oscillations in 1:1 resonance. This intermediate energy interval, the boundaries of which having been analytically identified, ${ }^{2}$ lies between the low and high energy regimes where different targeted energy transfer mechanisms from the directly forced LO to the NES arise. In order to track these different NES absorption mechanisms, the time evolution of the energy dissipated by the nonlinear attachment is computed by means of the energy dissipation measure $E_{N E S}(t)$, defined as

$$
E_{N E S}(t)=\frac{\lambda_{2} \int_{0}^{t}[\dot{z}(\tau)-\dot{x}(\tau)]^{2} d \tau}{\left(X^{2} / 2\right)} \times 100 .
$$


As shown by the expression (5), the measure $E_{N E S}(t)$ is normalised with respect to the initially imparted input energy.

In Figure 2, results obtained when the primary oscillator of the lightly damped system is impulsively excited at intermediate (a) and high (b) energy levels are reported. The response of the system is shown in terms of NES (red) and LO (blue) displacement time-histories. These two regimes were analytically addressed and interpreted in Ref. 1 and are here summarized for the sake of completeness. In particular, case (a) was tackled through an asymptotic analytical approach showing that the energetic regime of intensive energy transfer from the LO to the NES is effectively described in terms of LPTs. These are special periodic orbits of the underlying Hamiltonian system corresponding to maximum energy exchange (in the form of nonlinear periodic beats) between the LO and the NES. The solutions corresponding to case (b), albeit not directly shown in Ref. 1, corresponded to stationary states in an appropriately defined reduced phase plane and are nonlinear normal modes (NNMs) where the amplitude of the oscillation of the NES was twice that of the linear oscillator. Below each timehistory shown in Figure 2, the associated measures of
$E_{N E S}(t)$ are also depicted together with the evolution of the potential, kinetic, and dissipated energy components of the LO and the NES. Dashed lines represent the two stable equilibrium points, whereas the gray vertical bands highlight the time intervals of intense energy exchange from the linear oscillator to the non-linear attachment.

In order to compare the different evolutions of the energy dissipated by the NES in the different energy regions, the same time span of 50 time units is considered in the reported simulations. In Figure 2(a), the nonlinear beats (LPT) occurring in the intermediate energy region $(X=0.1)$ imply a regular intermittent sequence of strong energy exchange intervals; the amount of energy dissipated by the NES in the considered time interval is around $45 \%$. In Figure 2(b), the energy exchange due to $1: 1$ resonant capture, occurring in the high energy region $(X=0.55)$, is shown to take place at an almost constant, slower rate; the amount of energy dissipated by the NES in the considered time interval is around $8 \%$.

The low-energy main energy transfer mechanisms that will be addressed in this work are depicted in Figure 3. In particular, in Figure 3(a), the aperiodic alternating in-well

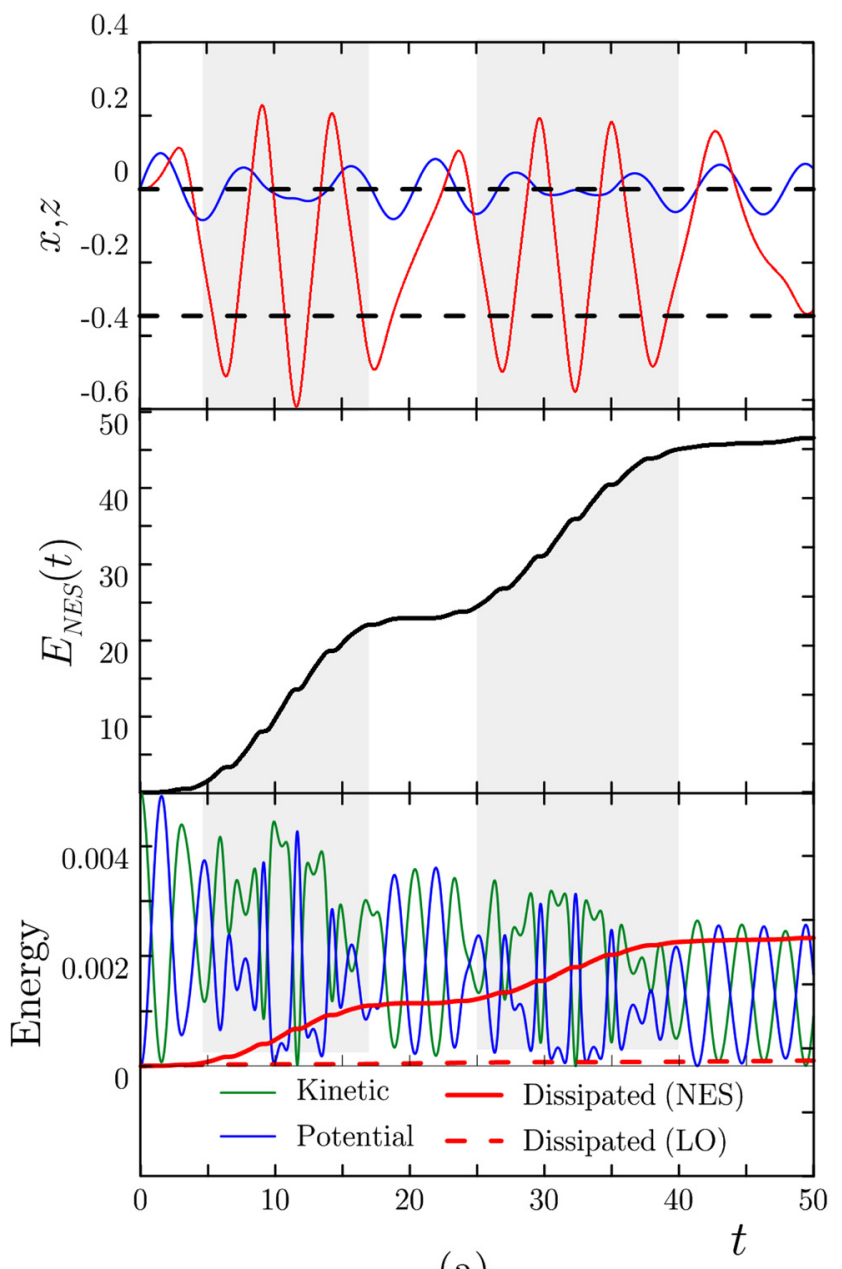

(a)

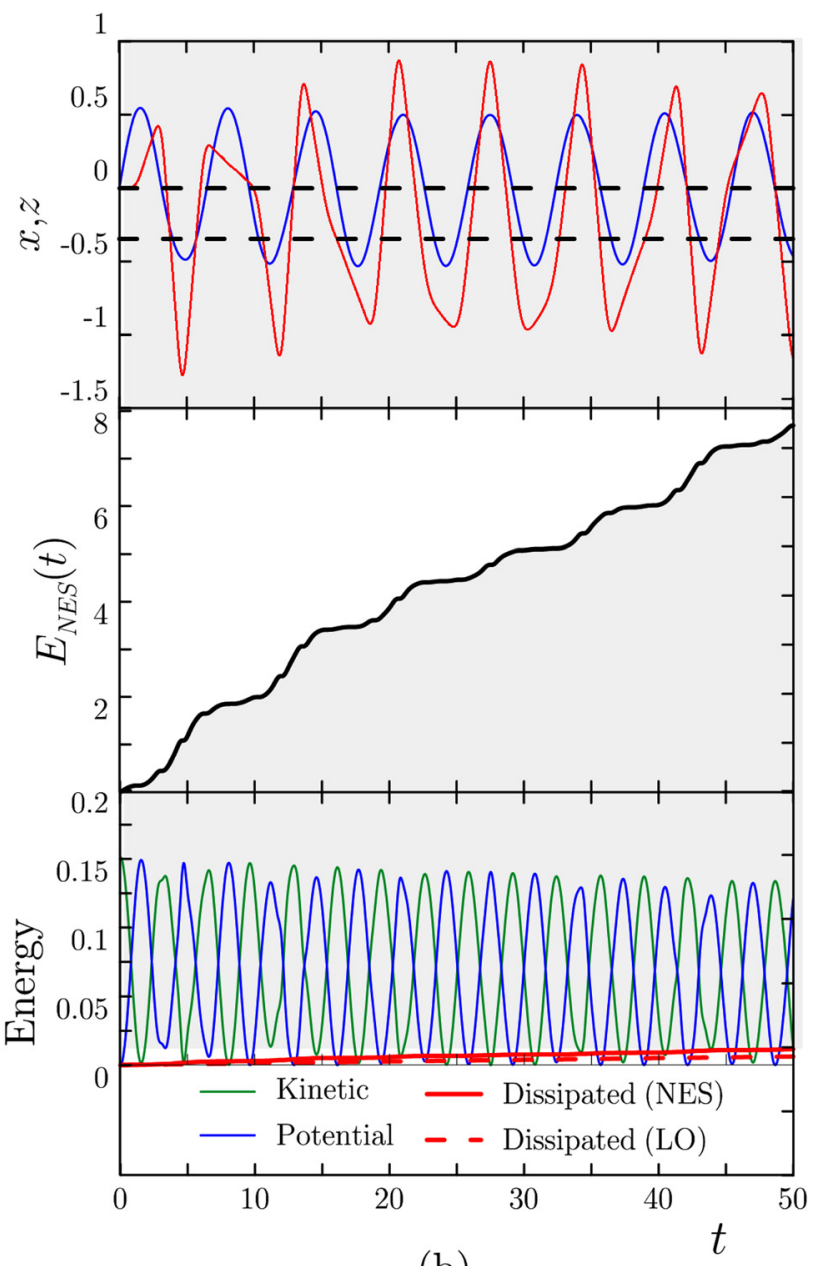

(b)

FIG. 2. Main energy transfer mechanisms at intermediate and high energy levels; NES (LO) displacement is in red (blue) and dashed lines represent the two stable equilibrium points. Parameters are: $C_{0}=-0.03, C=1.0, \lambda_{1}=\lambda_{2}=0.001$ : (a) nonlinear beats (LPT) occurring for intermediate input energy level $\left(X=0.1\right.$ ), associated $E_{N E S}(t)$ and energy components evolution; (b) 1:1 resonant capture (NNM) occurring for high input energy level ( $\left.X=0.55\right)$; the associated measures of $E_{N E S}(t)$ and the evolutions of the instantaneous energy components of the LO and the NES are also depicted. 


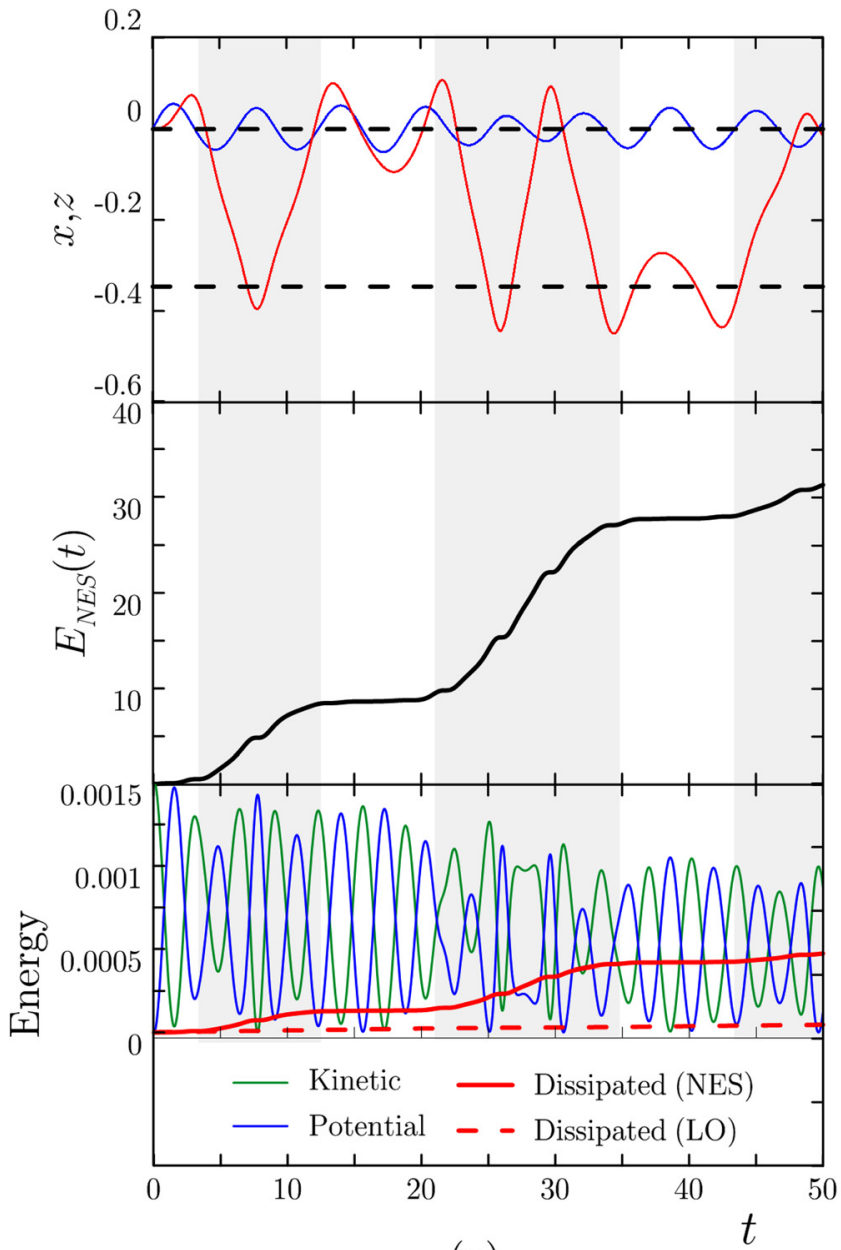

(a)

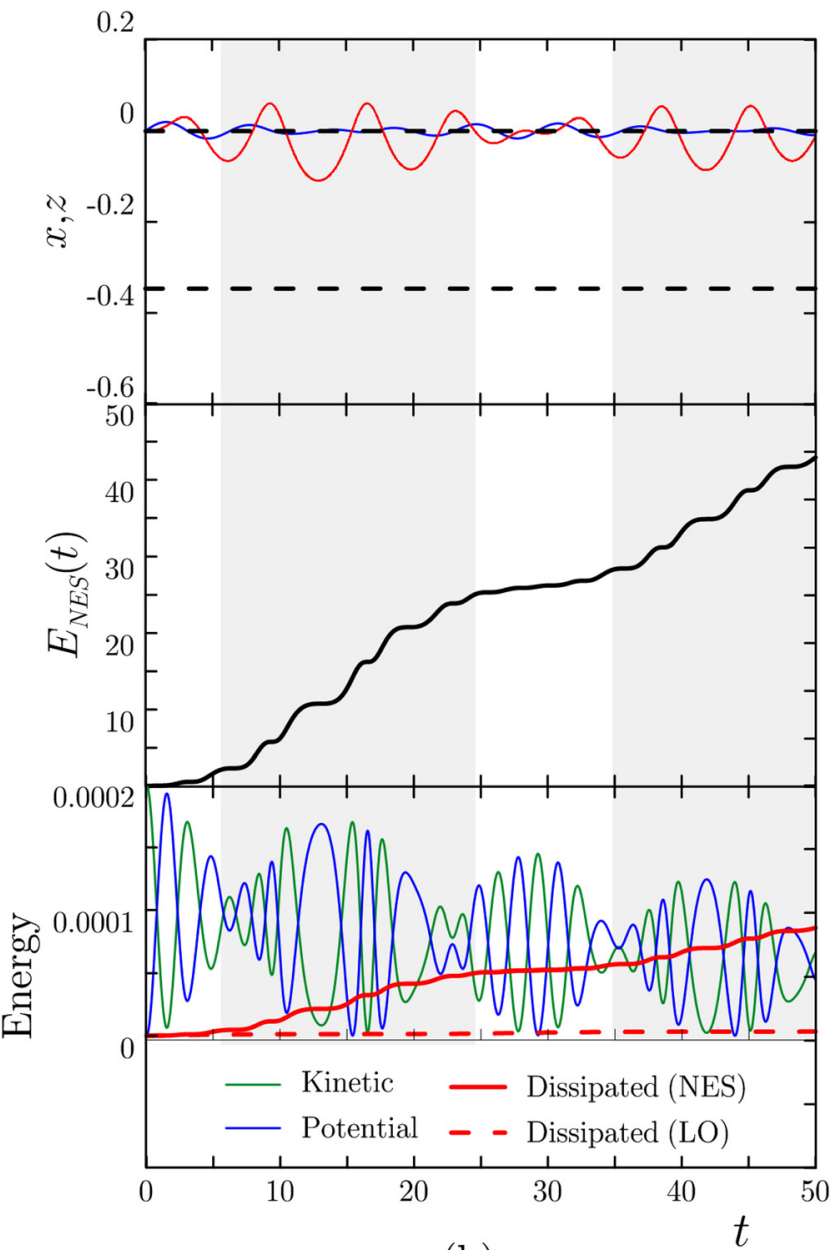

(b)

FIG. 3. Main energy transfer mechanisms at low energy levels; NES (LO) displacement is in red (blue) and dashed lines represent the two stable equilibrium points. Parameters are: $C_{0}=-0.03, C=1.0, \lambda_{1}=\lambda_{2}=0.001$ : (a) Aperiodic alternating in-well and cross-well oscillations occurring for low input energy level $(X=0.055)$, associated $E_{N E S}(t)$ and energy components evolution; (b) in-well nonlinear beats (secondary LPT) occurring for low input energy level $(X=0.02)$; the associated measures of $E_{N E S}(t)$ and the evolutions of the instantaneous energy components of the LO and the NES are also depicted.

and cross-well oscillations, obtained for $X=0.055$, show irregular (in both duration and time of occurrence) intermittent intervals of strong energy exchange; the amount of energy dissipated by the NES in the considered time interval is around 35\%. In Figure 3(b), these time intervals occur once again in a regular intermittent sequence corresponding to the secondary nonlinear beats (LPT) occurring at the very low energy region $(X=0.02)$; the amount of energy dissipated by the NES in the considered time interval is around $45 \%$.

\section{LOW-ENERGY TRANSFERS BASED ON PSEUDO-SEPARATRIX CHAOS}

In this section, the general features of the dynamics observed for low energy impulses acting on the LO are discussed. The reported numerical results aim to show that the two nonlinear targeted energy transfer mechanisms occurring at low energy shown in Figure 3 can be effectively described by reducing the dynamics to a single degree of freedom oscillator.

Towards this end, we reconsider the equations of motion (1) and introduce a new normalization of the system parameters and variables that will facilitate our study of cross-well oscillations. By introducing the new variable $u=v-x$ and rescaling the parameters of system (1) as follows:

$$
\begin{gathered}
\tau=\omega_{0} t ; \quad \varepsilon^{2}=\frac{m_{2}}{m_{1}} \ll 1 ; \quad \omega_{0}=\sqrt{\frac{k_{1}}{m_{1}}} ; \quad \bar{C}_{0}=\frac{k_{2}}{m_{2} \omega_{0}^{2}} ; \\
\bar{C}=\frac{k_{3}}{m_{2} \omega_{0}^{2}} \quad \mu_{1}=\frac{c_{1}}{m_{1} \omega_{0}} ; \quad \mu_{2}=\frac{c_{2}}{m_{2} \omega_{0}},
\end{gathered}
$$

the equation of motion (1) can be put in the form

$$
\begin{aligned}
& \left(1+\varepsilon^{2}\right) \ddot{x}+\varepsilon^{2} \ddot{u}+\mu_{1} \dot{x}+x=0, \\
& \ddot{u}+\ddot{x}+\mu_{2} \dot{u}+\bar{C}_{0} u+\bar{C} u^{3}=0,
\end{aligned}
$$

where the derivatives with respect to $\tau$ are considered. The initial conditions at $\tau=0$ are given by $x=u=0, \dot{x}=v_{0}$, $\dot{u}=-v_{0}$, and the mass ratio is kept fixed throughout this study at the value $\varepsilon^{2}=0.05$.

At first, in order to describe the influence of the negative stiffness on the relative displacement $u$, the evolution of the average frequency content of $u$ as the negative stiffness $C_{0}$ increases is considered. This global index is computed by 
taking a sequence of Discrete Fourier Transforms (DFT) of the time histories of the response $u$ corresponding to twenty values of $C_{0}$ between 0 and -1 . Since the signals are highly nonstationary, the spectral content obtained through the DFT provides an estimate of the average frequency content of the signals in time. The contour plots of the obtained surfaces are shown in Figures 4(a) and 4(b) for the undamped and damped cases $\left(\mu_{1}=\mu_{2}=0.01\right)$, respectively. For the low energy impulse $\left(v_{0}=0.25\right)$, the pure cubic configuration (i.e., $\left.C_{0}=0\right)$ shows a monochromatic response $(\omega=1)$ corresponding to the absence of energy transfer. As the negative $C_{0}$ increases, however, the spectral content of $u$ broadens for both the undamped and damped cases. In the latter case, close to $C_{0}=-1.0$, the frequency content spreads over the range of $0<\omega<\pi / 4$; the observed broad frequency content does not show any preferential frequency of oscillation of the NES, thereby suggesting that the cross-well response becomes aperiodic. Based on this first evidence of irregular oscillations, more insights into this dynamics are sought by considering the projection of the transient dynamics on the phase plane depicting the relative displacement $u$ versus its derivative $\dot{u}$.

At this point, we note that the potential energy of the system (4) is given by

$$
V(x, u)=\frac{1}{2} x^{2}+\frac{1}{4} \bar{C} u^{4}+\frac{1}{2} \bar{C}_{0} u^{2} .
$$

At the initial instant $\tau=0$, the potential energy is equal to zero (for $x_{0}=0$ and $v_{0}=0$ ), and the kinetic energy has its maximal value due to the impulsive nature of the applied excitation. However, we note that this condition can also be achieved at any instant when the condition $V(x, u)=0$ is satisfied, namely, for

$$
x= \pm u \sqrt{\frac{-2 \bar{C}_{0}-\bar{C} u^{2}}{2}} .
$$

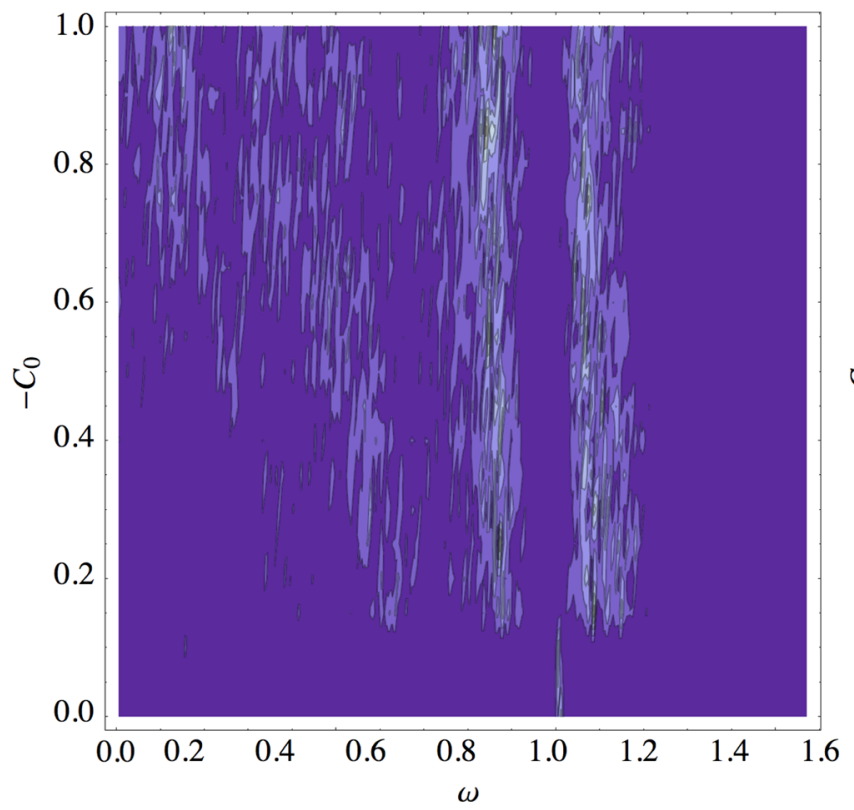

(a)
Clearly, this may occur not only when $x_{0}=0$ and $v_{0}=0$ but also whenever Eq. (9) is satisfied. So, this curve is a geometric locus of points in configuration space where the kinetic energy is maximal. If the phase space and, in particular, its projection into the plane $u-\dot{u}$, is considered, the kinetic energy is maximal for almost horizontal trajectories which can arise in the presence of a separatrix. Therefore, such trajectories have to be concentrated in the vicinity of the curve given by (9) which will be named as the pseudo-separatrix. This equipotential curve is represented by the black curve shown in Figure 5 (bottom) where the concentration of trajectories is also clearly depicted. The time-histories of the damped system's response and corresponding $u-\dot{u}$ phase-plane are also shown in Figure 5. As shown in this figure, through the projection on the phaseplane, outer (a), inner (c), and crossing (b) solutions can be conveniently classified with respect to the overlapped pseudo-separatrix belonging to the $u-x$ plane. The relative displacement $u$ (red) and the LO displacement $x$ (blue) are shown for three time intervals corresponding to different regimes; the dashed lines represent the two stable equilibrium points. Few initial nonlinear beats (LPTs), characterised by trajectories approaching the pseudo-separatrix and crossing it with a regular pace, can be observed (Figure 5(a)). Afterwards, as shown in Figure 5(b), as the energy decreases, aperiodic alternating cross-well and in-well oscillations take place. The latter entail irregular repeated pseudo-separatrix crossings, allowing for efficient energy absorption by the NES. The trajectories eventually enter (are captured by) one of the two wells and the dynamics of the relative displacement $u$ smoothly decays into one of the two stable equilibrium points. In essence, by depicting the low-energy dynamics in the phase-plane, it can be inferred that an equivalent single-degree-of-freedom nonlinear oscillator can be derived to capture and predict the two

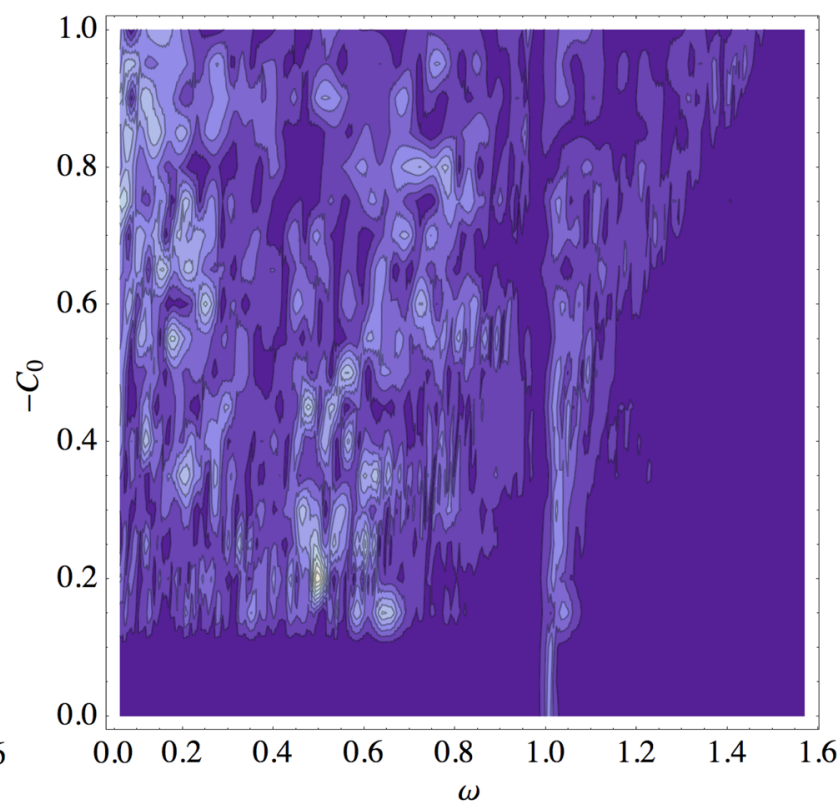

(b)

FIG. 4. Evolution of the average frequency content of $u$ as the negative stiffness $C_{0}$ increases. (a) Undamped case; and (b) damped case, $\mu_{1}=\mu_{2}=0.01$. 


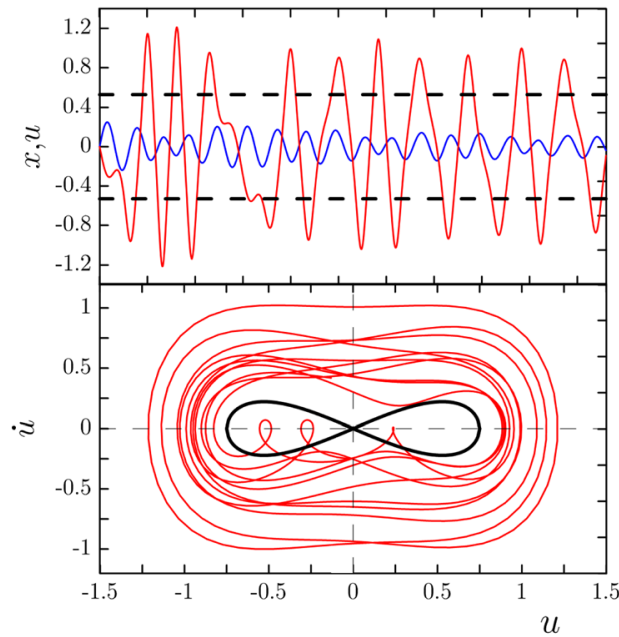

(a)

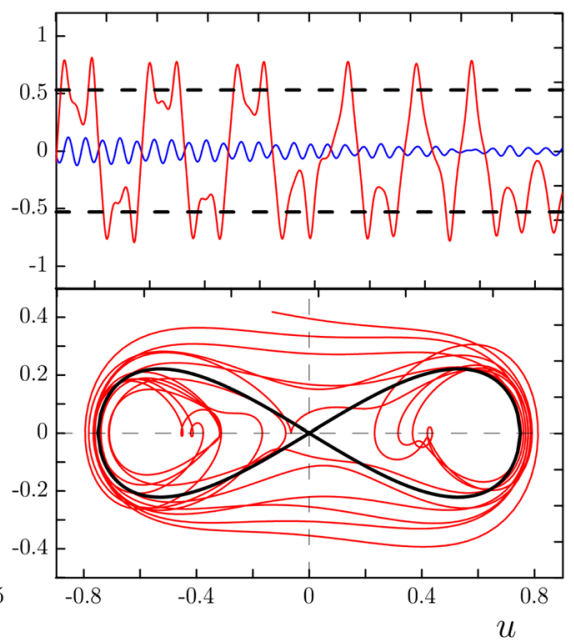

(b)

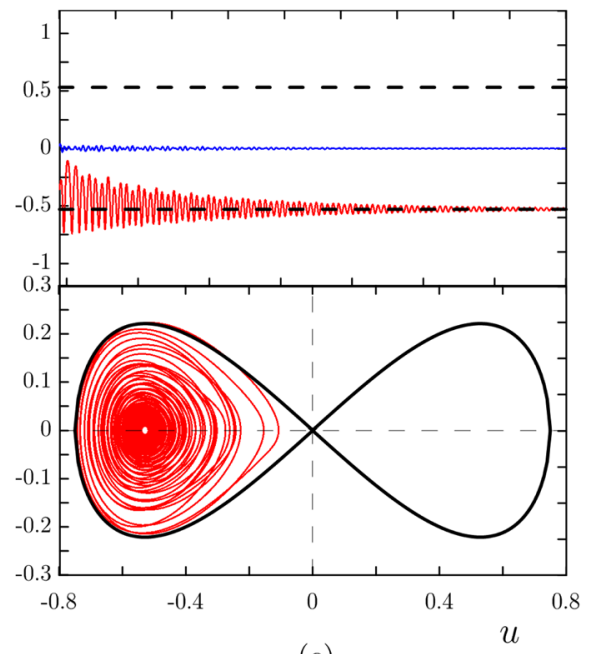

(c)

FIG. 5. Time-histories of the damped system's response and corresponding $u$ - $\dot{u}$ phase-plane. Relative displacement $u$ (red), LO displacement $x$ (blue); the dashed lines represent the two stable equilibrium points. The pseudo-separatrix on the $u-x$ plane is shown in black.

phenomena we are interested in, namely, the aperiodic cross-well oscillations and the in-well nonlinear beats.

\section{APERIODIC (CHAOTIC) CROSS-WELL OSCILLATIONS}

The analytical description of the aperiodic cross-well oscillations anticipated in Sections II and III is pursued by reducing the Eq. (5) to a single degree of freedom system. By assuming that $k_{2}$ is small, and that $x$ is much smaller than $u$, the renormalisation given by $\tilde{u}=u /\left(\varepsilon u_{0}\right), \tilde{x}=x / x_{0}$ enables one to rewrite (4) as follows (where the tilde in the normalized displacements is omitted from here on):

$$
\begin{array}{r}
\left(1+\varepsilon^{2}\right) \ddot{x}+\varepsilon^{3} \frac{u_{0}}{x_{0}} \ddot{u}+\varepsilon^{2} \mu_{1} \dot{x}+x=0, \\
\ddot{u}+a \ddot{x}+\mu_{2} \dot{u}+\bar{C}_{0} u+B u^{3}=0,
\end{array}
$$

where a lightly damped primary structure is assumed and

$$
\begin{aligned}
& \frac{x_{0}}{u_{0}}=O(\varepsilon) ; \quad a=\frac{x_{0}}{\varepsilon u_{0}}=O(1) ; \\
& B=\varepsilon^{2} u_{0}^{2} \bar{C}=O(1) ; \quad \bar{C}_{0}=O(1) .
\end{aligned}
$$

By retaining only the first order terms in Eq. (10), the following approximate system is obtained:

$$
\ddot{x}+x=0, \quad \ddot{u}+\mu_{2} \dot{u}+\bar{C}_{0} u+B u^{3}=-a \ddot{x} .
$$

From the solution of the first equation, namely, $x=A \sin (\tau+\varphi)$, we obtain the reduction of the full system to the single degree of freedom equation given by

$$
\ddot{U}+\mu_{2} \dot{U}+\bar{C}_{0} U+B U^{3}=-a A \sin (\tau+\varphi) .
$$

By rescaling Eq. (13) according to $\tau_{1}=\sqrt{B} \tau, \omega=1 / \sqrt{B}$, for $\bar{C}_{0} / B \simeq-1, \mu=\mu_{2} / B$, the SDOF system can be rewritten as

$$
\ddot{U}+\mu \dot{U}-U+U^{3}=A_{1} \sin (\omega \tau)+A_{2} \cos (\omega \tau),
$$

in which the derivatives are taken with respect to the new rescaled time variable $\tau_{1}$. Figure 6 depicts a comparison between the full (red) and the reduced (blue) system responses. The qualitative good agreement between the two systems can be observed in terms of both response time-histories and phase-plane orbits in which the pseudoseparatrix on the $u-x$ plane is also shown in black; the parameters adopted in the simulations are $B=0.6, \bar{C}_{0}$ $=-0.6, \mu_{2}=0.001$.

The reduction of the full system to a single oscillator governed by Eq. (14) in order to interpret the low-energy dynamics implies the possibility of exploiting classical results available for the forced, damped Duffing equation. The interest lies in predicting the occurrence of aperiodic cross-well oscillations taking place for energy intervals bounded above by LPTs and bounded below by in-well oscillations. Therefore, by considering Eq. (14), these lower and upper energy thresholds can be identified through the analytical approximations provided by the Melnikov approach and the Lyapunov characteristic exponents, respectively.

\section{A. Melnikov analysis for homoclinic bifurcation}

Starting from the analysis of the transition from the cross-well aperiodic oscillations to the in-well periodic ones, the Melnikov argument can be used to find the necessary conditions for the occurrence of the homoclinic bifurcation of the hilltop saddle. As known, this global approximate method aims at studying the persistence of periodic (resonant) and homoclinic orbits of a conservative system under weak perturbations provided by damping and external excitation. $^{18,19}$

We consider the following perturbed damped first order system derived from Eq. (14) by setting $U=x_{1}, \dot{U}=x_{2}$ :

$$
\dot{x}_{1}=x_{2} \quad \dot{x}_{2}=x_{1}-x_{1}^{3}+\epsilon\left[A \cos (\omega \tau)-\mu x_{2}\right] .
$$

In (15), $\epsilon$ is a small parameter governing the weak perturbation, while $A$ and $\mu$ represent amplitude of the harmonic excitation, directly related to the full system LO displacement, and damping, respectively. For $\epsilon=0$, the orbits of this system along the homoclinic loops (i.e., the separatrix) are 


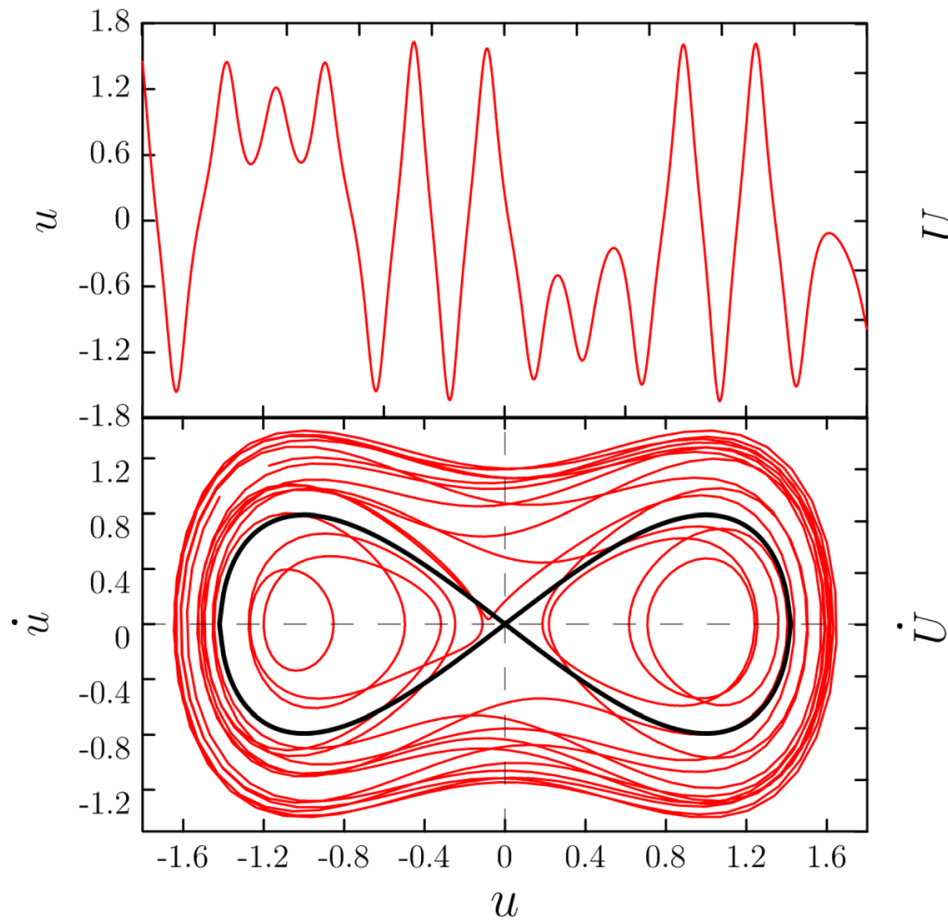

(a)

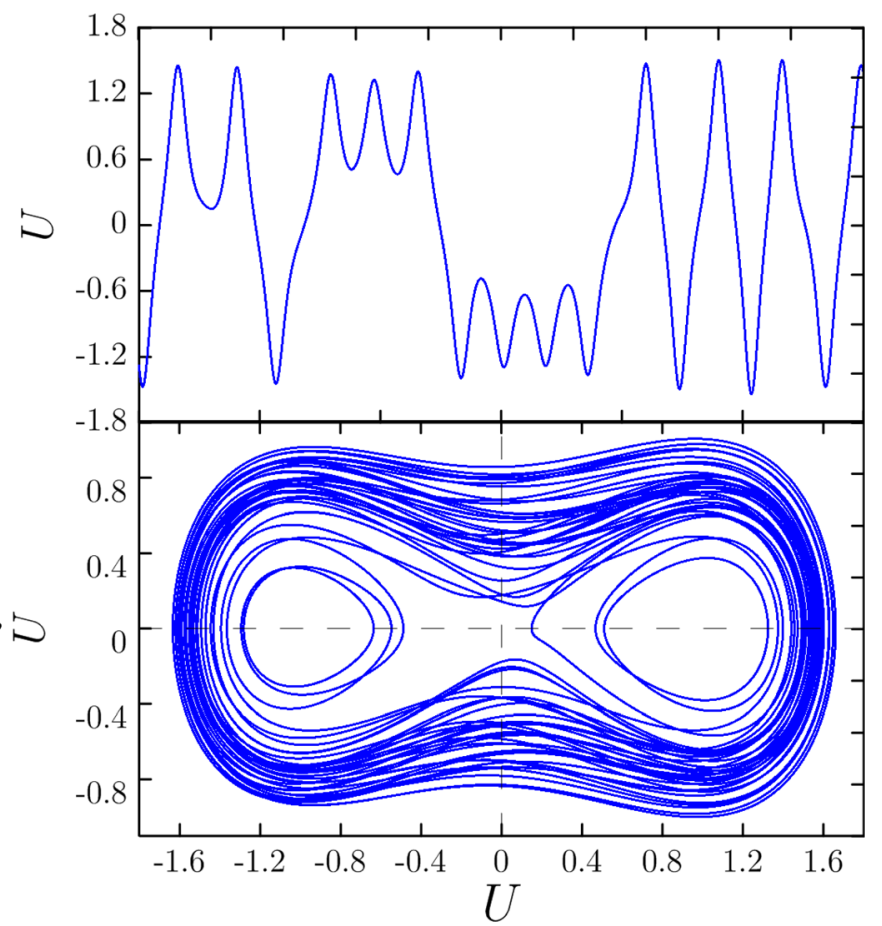

(b)

FIG. 6. Comparison between the full (red) and the reduced (blue) system responses. (a) Relative displacement of the full system (top) and corresponding orbit in a projection of the phase space (bottom); (b) relative displacement of the reduced system (top) and corresponding orbit in a projection of the phase space (bottom).

denoted as $x_{1, h}, x_{2, h}$ and can be analytically derived by quadratures. Then, the homoclinic Melnikov function describing the distance between the perturbed stable and unperturbed manifolds of the weakly perturbed system with $\epsilon$ not equal to zero is defined as

$$
M\left(\tau_{0}, \phi_{0}\right)=\int_{-\infty}^{\infty}-\mu x_{2, h}^{2} \pm A x_{1, h} \cos \left[\omega\left(\tau+\tau_{0}\right)+\phi_{0}\right] d t .
$$

Given that $x_{1, h}= \pm \sqrt{2} \operatorname{sech} \tau, x_{2, h}=\mp \sqrt{2} \operatorname{sech} \tau \tanh \tau$, Eq. (16) becomes

$$
M\left(\tau_{0}, \phi_{0}\right)=-\frac{4}{3} \mu \pm \sqrt{2} A \pi \omega \operatorname{sech} \frac{\pi \omega}{2} \sin \omega \tau_{0}+\phi_{0} .
$$

A necessary condition for a homoclinic bifurcation to occur is obtained by the inequality ${ }^{18,19}$

$$
A<\frac{\frac{4}{3} \mu}{\sqrt{2} \pi \omega \operatorname{sech} \frac{\pi \omega}{2}},
$$

from which the critical amplitude $A_{c r}$ is determined when (18) becomes an equation. It can then be concluded that for $A>A_{c r}$ the stable and the unstable manifolds intersect transversely leading to homoclinic bifurcations and chaotic trajectories, while they are disjoint for $A<A_{c r}$. For $A=A_{c r}$, the stable and unstable manifolds are tangent to each other, and this transition from detachment to intersection through a tangency of manifolds is asymptotically studied. The function $A_{c r}(\omega)$ is shown in Figure 7(a) for three values of damping ( $\mu=0.01,0.02,0.03)$. The vertical dashed line corresponds to the value $\omega=1 / \sqrt{B}$ for $B=0.6$ considered for the numerical simulations shown in Figures 7(b)-7(d) corresponding to $A_{3}$ $=0.15, A_{2}=0.05, A_{1}=0.009$, respectively, with $\mu=0.01$. As predicted by the analytical approximation, as the amplitude of the harmonic forcing increases, the threshold is reached and aperiodic (chaotic) cross-well oscillations occur.

The full system governed by Eqs. (10) is now reconsidered in order to relate the critical amplitude of the harmonic forcing acting on the reduced single oscillator to the amplitude of the LO displacement $x$. In particular, it is shown that the critical value $A_{c r}$ provides a reliable estimate of the critical LO displacement leading to the transition from the crosswell aperiodic (chaotic) oscillations to the periodic in-well ones in terms of the relative displacement $u$ between LO and NES. The numerical results reported in Figure 8 refer to the response of system (15) where $\epsilon^{2}=0.05, B=0.6$, $\bar{C}_{0}=-0.6, u_{0}=0.774, x_{0}=0.173$. The green and red amplitude intervals of the LO response $x$ highlighted in Figure 8(c) correspond to the forcing amplitude regions reported in Figure 7(a) for the single-degree-of-freedom reduced order system. Furthermore, the vertical dashed line reported in Figures 8(b) and 8(c) corresponds to the transition to the periodic in-well oscillations taking place when the amplitude of the LO response decreases and enters the red region $(|x|<0.05)$, in good agreement with the Melnikov prediction shown in Figure 7(a). Such periodic in-well oscillations show rather clearly the nonlinear beat phenomenon during which significant energy exchanges between the LO and the NES take place; this regime will be specifically addressed in Sec. V. 

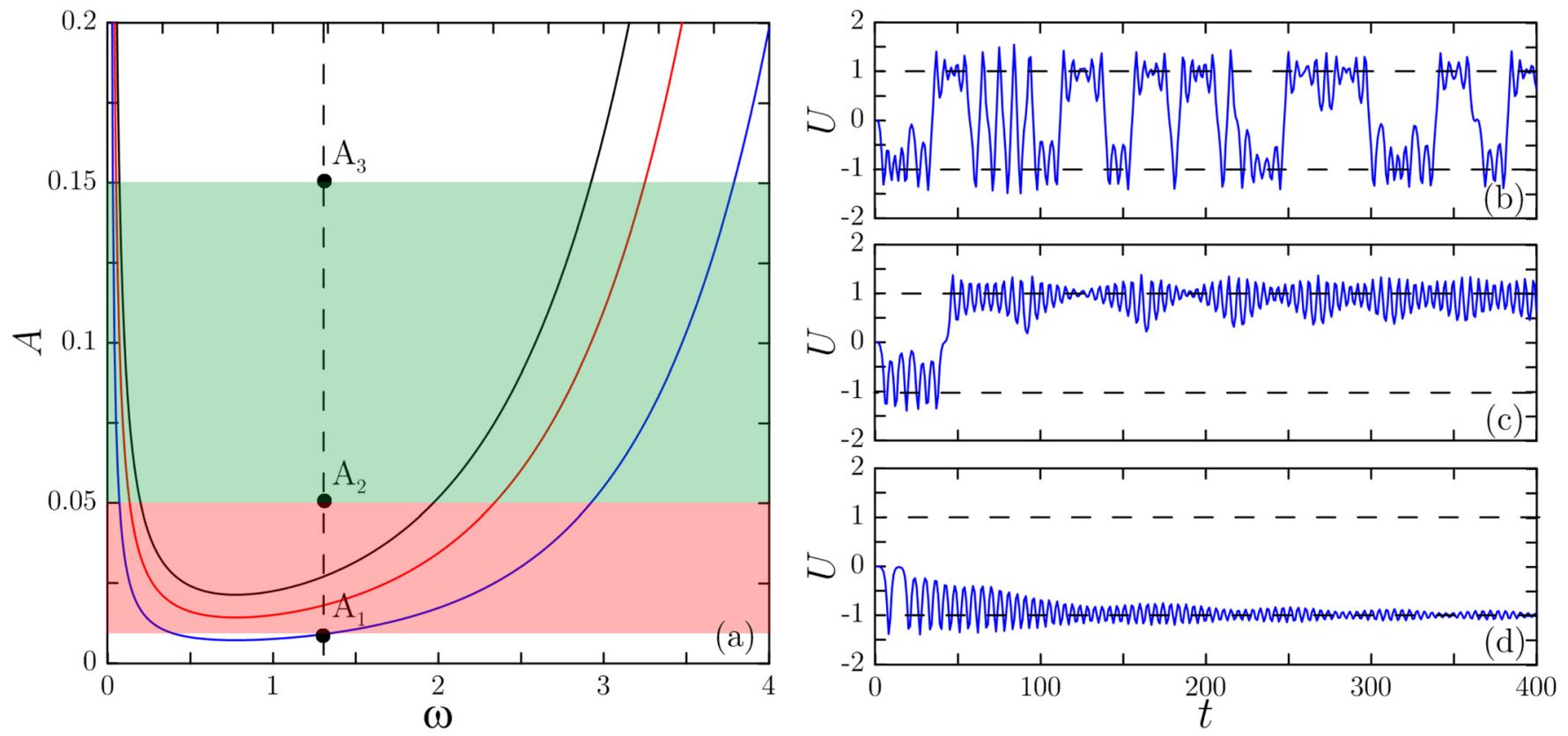

FIG. 7. Critical forcing amplitude $A_{c r}$ for different damping values and oscillator responses for $\mu=0.01$ : (a) $A_{c r}$ ( $\omega$ ) for $\mu=0.01$ (blue), $\mu=0.05$ (red), $\mu=$ 0.15 (black) derived by homoclinic Melnikov analysis; (b) oscillator cross-well aperiodic response for forcing amplitude $A_{3}=0.15$ by direct numerical integration of system (15); (c) transition from cross- to modulated in-well oscillator response for forcing amplitude $A_{2}=0.05$; and (d) oscillator in-well response for forcing amplitude $A_{1}=0.009$.

\section{B. Lyapunov characteristic exponents analysis}

The Melnikov analysis carried out in Section IV A enables one to identify an approximate lower bound of the LO displacement amplitude leading to the existence of aperiodic (chaotic) cross-well oscillations. In this section, the analysis is extended by computing the Lyapunov characteristic exponents in order to identify both lower and upper boundaries of the region of chaotic cross-well oscillations. As well known, the Lyapunov exponents (LEs) provide a quantitative measure of the degree of stochasticity of a trajectory, as they represent the mean exponential rate of divergence from it of trajectories. ${ }^{20}$

The LE is defined as

$$
\lambda_{L}=\lim _{d_{0} \rightarrow 0, t \rightarrow \infty}\left[\frac{1}{t} \ln \left(\frac{d(t)}{d_{0}}\right)\right],
$$

where $d(t)$ is the distance in phase space between a given orbit and a test orbit beginning with a nearby initial
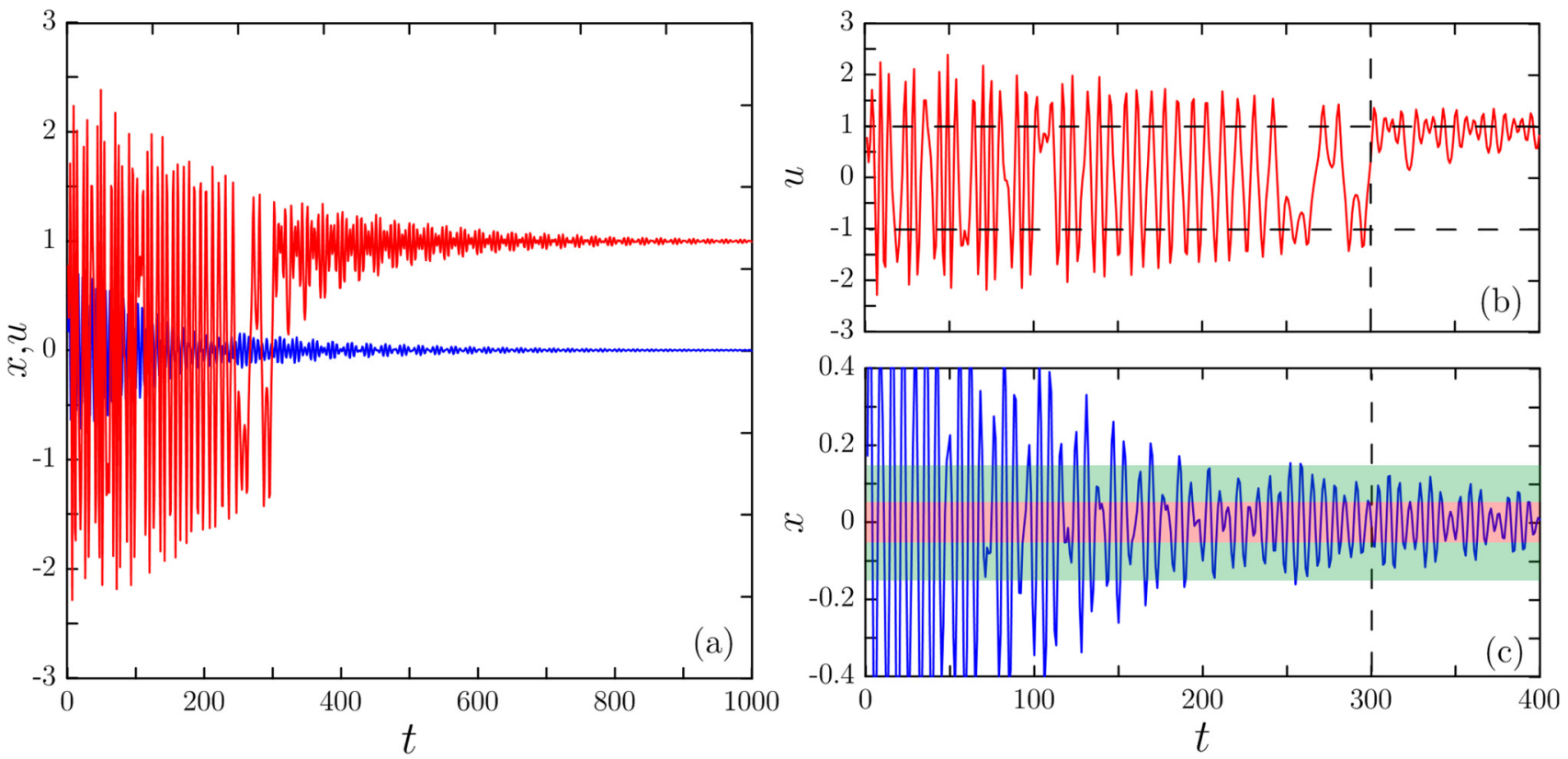

FIG. 8. Transient response of the full system (10): (a) Time-hystories of the LO displacement $x$ (blue) and the relative displacement $u$ (red); (b) closer view of $u$ during the transition (vertical dashed line) from aperiodic cross-well oscillations to in-well periodic secondary nonlinear beats; (c) closer view of $x$ during the same time interval highlighting the green and red amplitude regions corresponding to Figure 7(a) as predicted by the sdof Melnikov analysis. 
condition at a distance $d(0)=d_{0}$. There is one LE $\lambda_{L}$ for each phase space dimension in a general multi-dimensional dynamical system, and the maximum of this set is called the maximal Lyapunov exponent or the Lyapunov characteristic exponent. An analytical estimation of such characteristic exponents is usually impracticable due to the nonintegrability of the chaotic motion, so one must resort to numerical methods for its estimation. Among the numerous numerical schemes proposed for the computation of the Lyapunov characteristic exponent, the "renormalization" scheme developed by Benettin and collaborators is implemented here. ${ }^{21}$ According to this method, at first, an initial condition for the orbit and a second nearby test orbit at a distance $d_{0}$ are chosen. Then, the equations of motion for the original and test orbits are solved by renormalizing the latter at $t=\kappa \tau$, i.e., at integer multiples of $\tau$. At each $k$-multiple of $\tau$, the partial sum of the exponential growth rate for each segment given by

$$
\lambda_{k}=\frac{1}{k \tau} \sum_{j=1}^{k} \ln \left(\frac{d_{j}}{d_{0}}\right)
$$

is computed and it can be shown that the maximal Lyapunov exponent is obtained as $\lim _{k \rightarrow \infty} \lambda_{k}$. Therefore, for regular orbits, $\lambda_{k}$ will decrease toward zero as $k$ increases, while for chaotic orbits, $\lambda_{k}$ should approach a positive value as $k$ increases. In Figure 9, the reduced order system governed by the damped $(\mu=0.01)$ SDOF oscillator displacement time-hystories and the corresponding Lyapunov characteristic exponents are shown, for different magnitudes of the harmonic excitation: Regular orbits are obtained for $A=0.01$ (Figure 9(c)) and $A=0.26$ (Figure 9(a)), while chaotic orbits occur for $A=0.15$ (Figure 9(b)). In particular, as the amplitude increases, periodic in-well oscillations, aperiodic cross-well oscillations, and periodic cross-well oscillations take place. The lower bound obtained is in good agreement with the Melnikov approximation. Moreover, the linear oscillator displacement range of $0.01-0.26$ within which chaotic cross-well oscillations occur obtained through the Lyapunov characteristic exponents is in good agreement with the results obtained for the full system shown in Figure 8(c).

\section{LOW-ENERGY TRANSFERS BASED ON IN-WELL NONLINEAR BEAT PHENOMENA}

In this section, the low-amplitude nonlinear beats taking place once the NES dynamics falls in one of the two potential wells (see Figure 8(a)) are studied by interpreting them as LPTs. ${ }^{2}$ LPTs $^{22,23}$ correspond to regimes of most intense energy exchanges between different particles or nonlinear normal modes ${ }^{24}$ of a dynamical system, and their role in the theory of non-stationary resonant dynamics is similar to the role of NNMs in stationary resonant dynamics. Contrary, however, to stationary and non-stationary non-resonant oscillations described efficiently in the framework of NNMs, resonant non-stationary processes are characterized by strong modulations and intense energy exchanges between different parts or modes of a system. Hence, the concept of LPT will be important in the discussion of energy exchange phenomena caused by the essential stiffness nonlinearities of the system considered. An LPT is an orbit corresponding to zero initial conditions of the system, with the exception of the initial displacement of the LO.

Different from the case of the (primary) LPTs studied in Ref. 1 where a symmetric system was considered, here, the asymmetric case of LPTs must be analyzed. ${ }^{25}$ In order to describe this peculiar dynamical regime, it is convenient to refer to a Poincaré map of the original system, governed by
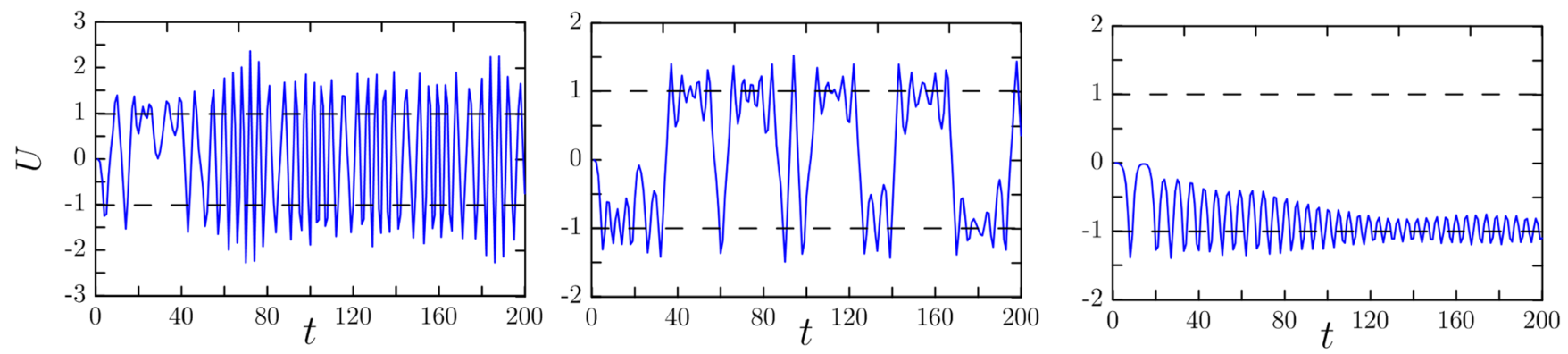

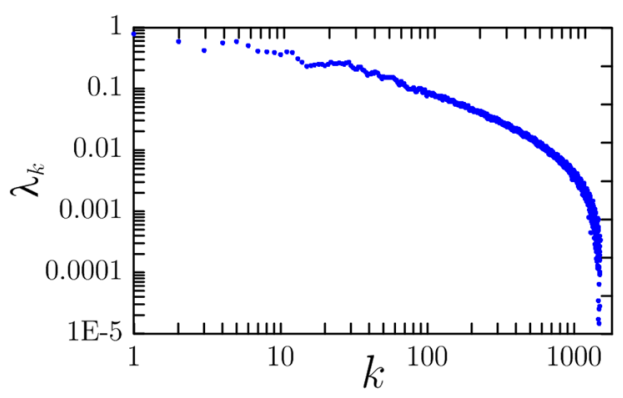

(a)

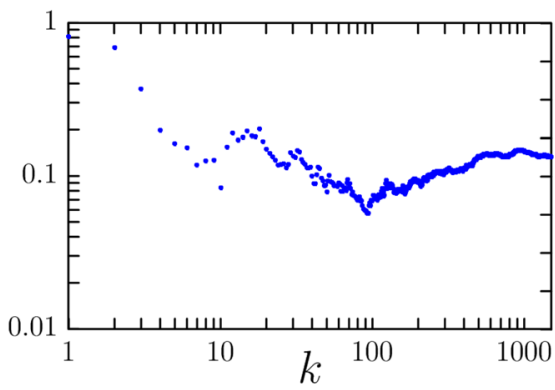

(b)

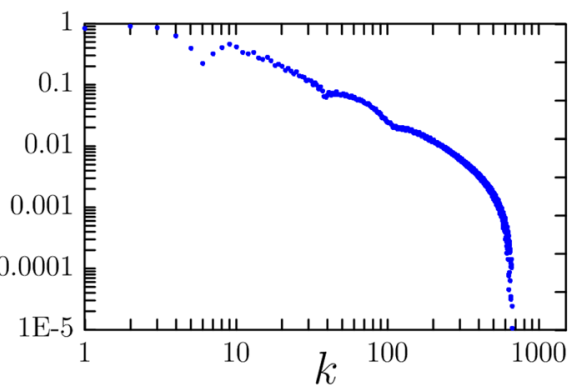

(c)

FIG. 9. Reduced damped ( $\mu=0.01)$ SDOF system displacement time-hystories (top) and corresponding Lyapunov characteristic exponents on log-log plots (bottom): (a) Regular orbit (periodic cross-well oscillations) for $A=0.26$; (b) chaotic orbit (aperiodic cross-well oscillations) for $A=0.15$; and (c) regular orbit (periodic in-well oscillations) for $A=0.009$. 
(1) expressed in non dimensional form, in the nonlinear oscillator phase space. ${ }^{2}$ Figures $10(\mathrm{~b})$ and 10(d) show the map obtained at intermediate (i.e., $E=0.006$ ) and low energy level (i.e., $E=1.0 \mathrm{E}-6$ ), respectively, representing the trajectories of the system for two sets of initial conditions: $\left\{x_{0}, v_{0}=0 ; \dot{x}_{0}, \dot{v}_{0} \neq 0\right\}$ and $\left\{x_{0}, v_{0} \neq 0 ; \dot{x}_{0}, \dot{v}_{0}=0\right\}$. The response corresponding to a primary LPT (studied in Ref. 2) is shown in Figure 10(a) and its trajectory is highlighted in the Poincaré map shown in Figure 10(b). In Figures 10(c) and 10(d), the considered energy level is lower than the energy barrier and the phase space is characterized by two lobes of chaotic orbits including islands of regular motion. The two symmetric islands of regular motion are filled with quasi-periodic orbits encircling two stationary points corresponding to dynamics in 1:1 resonance of oscillations of the LO and in-well oscillations of the NES. The trajectories separating the regions of regular motion encircling these stationary points are the secondary LPTs triggering the low amplitude, nonlinear beats in which we are interested as shown in Figure 10(c).

The reduced system governing the in-well dynamics is now given by

$$
\ddot{U}+\mu \dot{U}+c_{1} U+c_{2} U^{2}+c_{3} U^{3}=F \sin (\omega \tau+\varphi) .
$$

So, Eq. (21) replaces system (14) for the dynamics evolving in the neighbourhood of the two stable equilibria with parameters given by

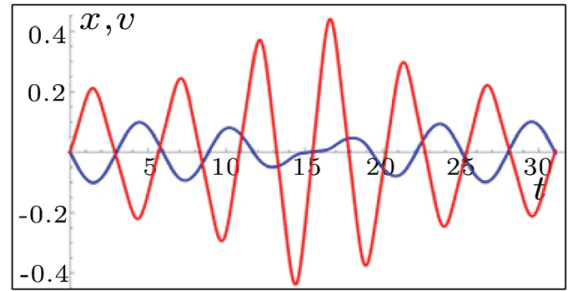

(a)

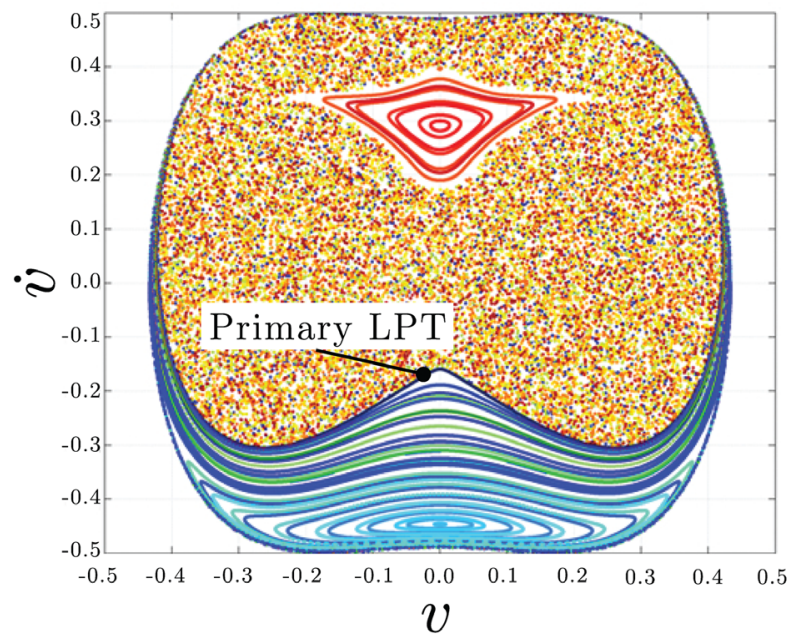

(b)

$$
\begin{gathered}
c_{1}=\frac{\bar{C}_{0}+3 \bar{C} d}{B} ; \quad c_{2}=\frac{D}{B} ; \quad c_{3}=1 ; \\
\mu=\frac{\mu_{2}}{B} ; \quad F=-\frac{a A}{B},
\end{gathered}
$$

where the $O(1)$ coefficient $D=3 \bar{C} d \varepsilon u_{0}$ has been introduced. Equation (21) can be rescaled as

$$
\ddot{u}+\mu \varepsilon^{2} \dot{u}+u+4 \alpha \epsilon u^{2}+8 \beta \epsilon^{2} u^{3}=2 \epsilon^{2} F \sin \left(\frac{\omega}{\omega_{0}} \tau\right),
$$

in which

$$
\begin{aligned}
& \tau_{0}=\omega_{0} \tau ; \quad \omega_{0}=\sqrt{c_{1}} ; \quad \alpha=\frac{c_{2}}{\varepsilon 4 c_{1}} ; \\
& \beta=\frac{c_{3}}{\varepsilon^{2} 8 c_{1}} ; \quad \tilde{\mu}=\frac{\mu}{\varepsilon^{2} \sqrt{c_{1}}} ; \quad \tilde{F}=\frac{F}{2 \varepsilon^{2} c_{1}},
\end{aligned}
$$

and the tildes in the forcing amplitude $F$ and damping parameter $\mu$ were omitted. By denoting $\dot{u}=v$ and applying complexification, the variables $\psi=v+j u$ and $\psi^{*}=v-j u$ are introduced. Then, as proposed in Ref. 25 and reported in detail in the Appendix, through the multiple scale method the main asymptotic approximation $\varphi_{0}$ of the complex modulation $\varphi$, through which the complex variables are expressed, is derived. At the slow time scale $\tau_{2}=\varepsilon^{2} \tau$, by denoting $\varphi_{0}=a e^{j \delta}$ and introducing the detuning parameter $s$ such that $\omega / \omega_{0}=1+\varepsilon^{2} s$, the following system (see the Appendix for details) is obtained:

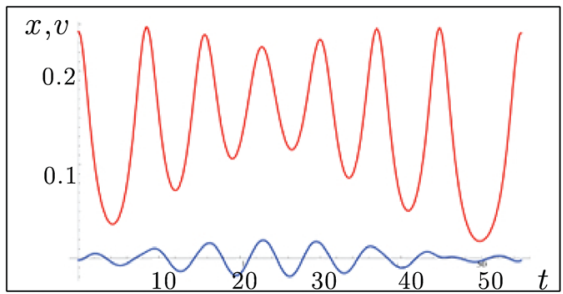

(c)

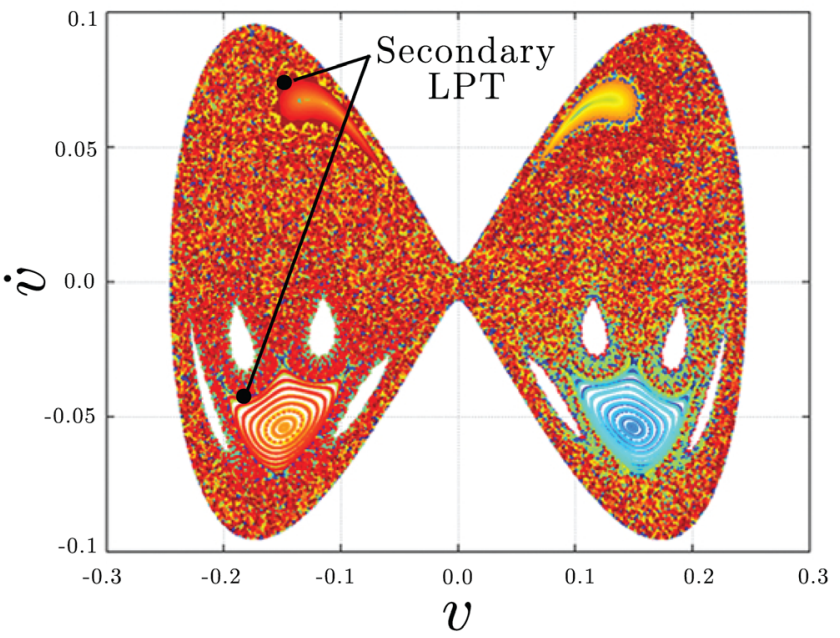

(d)

FIG. 10. Poincaré sections of the original system for $C_{0}=-0.03, C=1.0$, and associated responses, $v$ (red), $x$ (blue): (a) and (b) Primary LPT at intermediate energy level (i.e., $E=0.006$ ); (c) and (d) secondary LPT at low energy level (i.e., $E=1.0 \mathrm{E}-6$ ); (a) cross-well high amplitude nonlinear beat; (b)and (d) projection of the dynamics to the NES phase-space $v, \dot{v}$; and (c) in-well low amplitude nonlinear beat. 


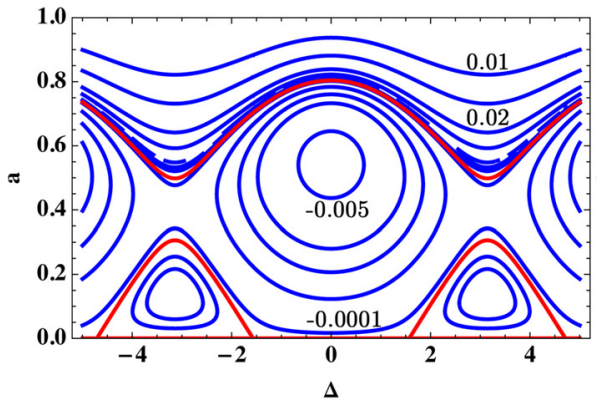

(a)

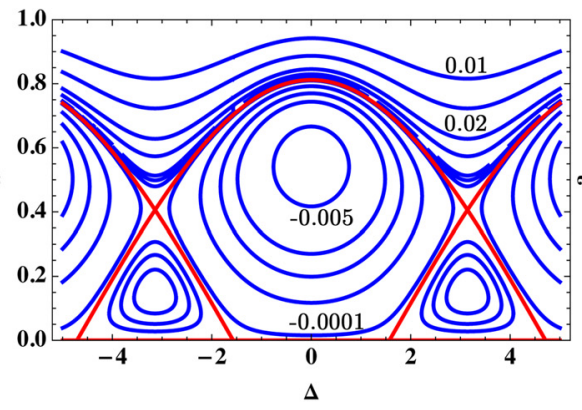

(b)

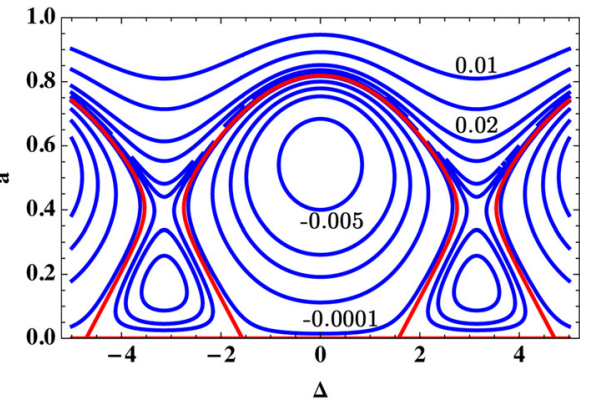

(c)

FIG. 11. Asymmetric trajectories on the phase plane for different values of the constant $C$, see Eq. (22), with limiting phase trajectories (red) corresponding to $C=0$ : (a) $\alpha^{*}<\alpha_{L P T}^{*}$; (b) $\alpha^{*}=\alpha_{L P T}^{*}$; and (c) $\alpha^{*}>\alpha_{L P T}^{*}$.

$$
\frac{d a}{d \tau_{2}}+\mu a=-F \sin \Delta, \quad a \frac{d \Delta}{d \tau_{2}}+a s-\alpha^{*} a^{3}=-F \cos \Delta,
$$

in which $\Delta=\delta-s \tau_{2}$. In (25), $\Delta$ characterises the phase shift between the LO and the NES, $a$ represents the envelope of the NES displacement, and $F$ characterises the amplitude of the LO. For the conservative case, the first integral of this system is given by

$$
H=\frac{1}{4} \alpha^{*} a^{4}-\frac{s a^{2}}{2}-F a \cos \Delta=C,
$$

in which $\alpha^{*}=3 \beta-\frac{20}{3} \alpha^{2}$ and $C$ is a constant depending on the initial conditions. From (26), the LPT corresponding to the contour obtained for $C=0$ can be depicted on the $a-\Delta$ plane and is represented by the curves passing through the segment $a=0$ as shown in Figure 11 (red curve). This orbit represents the most intense energy exchange between the LO and the NES for in-well oscillations, in the form of modulated nonlinear beats under condition of resonance (i.e., of commensurate ratio of the frequencies of the LO and the NES). Other curves associated with different initial conditions and corresponding to quasi-periodic orbits with less intense energy exchanges between the LO and the NES are also represented. The parameters considered in the simulation are $\alpha=0.205, \beta=0.153$ and the amplitude of the forcing term is $F=0.006$. This set of parameters corresponds to the case shown in Figure 8 in which the occurrence of nonlinear beats was clearly observed during in-well oscillations. In particular, in this approximation, a smaller detuning parameter $(s=0.044)$ is considered in order to be consistent with the asymptotic expansion implying a 1:1 resonance condition. The condition $H=0$ yields the parameter $\alpha^{*}$ corresponding to the transition between different types of LPT, given by

$$
\alpha_{L P T}^{*}=\frac{2 s^{3}}{27 F^{2}} \text {. }
$$

As shown in Figure 11(a), small oscillations occur for $\alpha^{*}<\alpha_{L P T}^{*}$ and the low amplitude LPT encircles the centre at $\Delta= \pm \pi$. In this case, the NES takes a relatively small amount of energy from the LO. For $\alpha^{*}=\alpha_{L P T}^{*}$, both elliptic and hyperbolic points appear on the LPT at $\Delta= \pm \pi$ (Figure 11(b)). At this threshold value, the LPT coincides with the separatrix and the time of energy transfer from the LO to the NES becomes ideally infinite. Afterwards, as shown in Figure 11(c) where $\alpha^{*}>\alpha_{L P T}^{*}$, large LPTs can be observed; in this case, the NES takes a relatively large amount of energy from the LO and this energy transfer occurs in a finite time interval. The phase plane shows that the LPT encircles the centre at $\Delta=0$ and its amplitude increases providing a clear indication of the occurrence of large nonlinear oscillations, namely, the secondary nonlinear beats.

As shown in Figure 12, the analytical prediction is numerically validated by integrating directly Eq. (23) for $\alpha^{*}$ $<\alpha_{L P T}^{*}$ (blue) and $\alpha^{*}>\alpha_{L P T}^{*}$ (red). The transition from small to large LPTs is accurately captured since $\alpha_{L P T}^{*}=0.179$ (it corresponds to the parameters considered for the results reported in Figure 11) and the two solutions shown in Figure 12 correspond to $\alpha^{*}=0.173$ (blue) and $\alpha^{*}=0.185$ (red).

For the conservative case, an alternative description of the LPTs can be derived by means of a second order differential equation. The latter is obtained by considering that for $H=0$

$$
\cos \Delta=\frac{\alpha^{*} a^{3}-2 s a}{4 F},
$$

so that, by differentiating the first equation in (25) with respect to $\tau_{2}$ and substituting the above expression for $\cos \Delta$, the following system is obtained:

$$
\frac{d^{2} a}{d \tau_{2}^{2}}=-F \cos \Delta \frac{d \Delta}{d \tau_{2}}, \quad \frac{d \Delta}{d \tau_{2}}=-\frac{s}{2}+\frac{3 \alpha^{*} a^{2}}{4},
$$

leading to the differential equation for the LPT amplitude

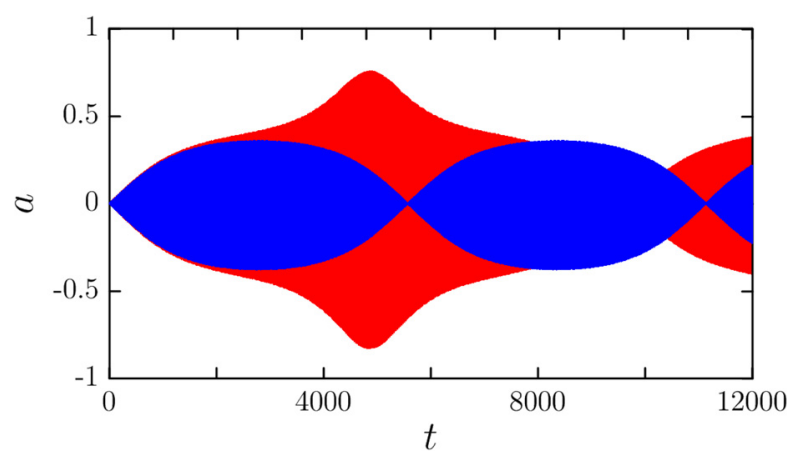

FIG. 12. Numerical validation of limiting phase trajectories corresponding to $\alpha^{*}<\alpha_{L P T}$ (blue) and $\alpha^{*}>\alpha_{L P T}$ (red). 


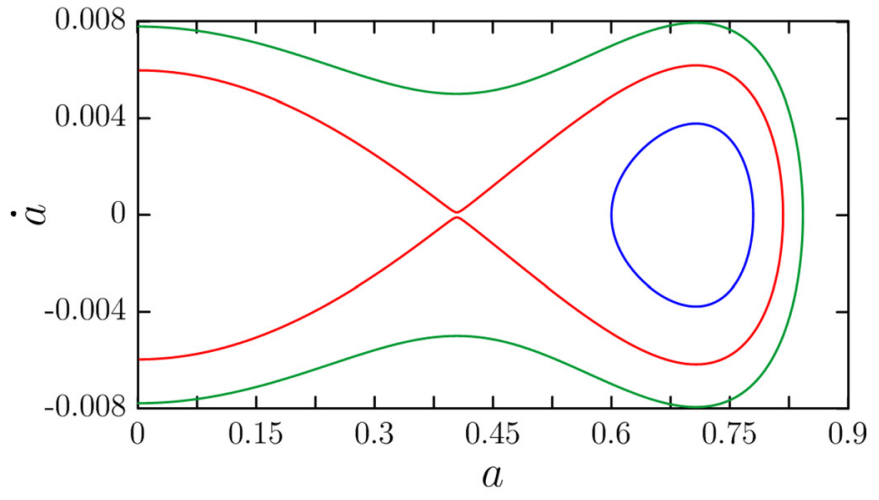

(a)

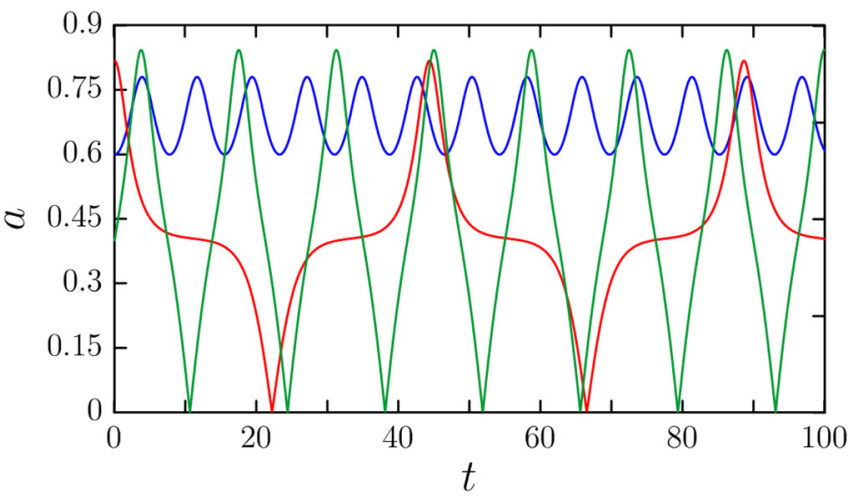

(b)

FIG. 13. Analytical approximation of limiting phase trajectories for $\mu_{1}=0.0005, \mu_{2}=0.004, \mu_{3}=0.006$ and initial conditions inside (blue), on (red), and outside (green) the separatrix. (a) Phase-plane; (b) time-history.

$$
\frac{d^{2} a}{d \tau^{2}}+\mu_{1} a-\mu_{2} a^{3}+\mu_{3} a^{5}=0
$$

where

$$
\mu_{1}=\frac{s^{2}}{4}, \quad \mu_{2}=\frac{s \alpha^{*}}{2}, \quad \mu_{3}=\frac{3 \alpha^{*^{2}}}{16} .
$$

Figure 13(a) shows the phase plane obtained by integrating Eq. (29) for $\mu_{1}=0.0005, \mu_{2}=0.004, \mu_{3}=0.006$. Three initial conditions are considered representing trajectories lying inside (blue), on (red), and outside (green) the separatrix. These three qualitatively different solutions correspond to the three cases reported in Figures 11(a)-11(c), respectively. During intense energy transfer, $\dot{a}$ takes large values and the trajectories corresponding to this fast motion tend to become straight lines on the phase plane. It must be emphasised that Figure 13(a) provides a dynamical representation of the LPTs which is merely related to in-well oscillations. Therefore, by considering the corresponding time-histories shown in Figure 13(b), an analytical estimate of the time duration of the transitions between the two wells can be inferred. In particular, by adopting the same parameters considered in the numerical results reported in Sec. II, the transition time intervals (around 6 time units) obtained for the large LPTs (green) are in good agreement with those shown in Sec. II (Figure 3(a)). Since during these transitions intense energy exchanges between the LO and the NES occur, the analytical description of the LPTs provided in this work can be used in a predictive capacity, that is, to design the parameters of the system for most intense targeted energy transfer from the directly excited LO to the bistable NES.

\section{CONCLUSIONS}

The low-energy transient dynamics of a bistable nonlinear energy sink coupled system is addressed. The system consists of a primary LO connected to a lightweight mass (NES) by means of a spring with both cubic nonlinear and negative linear components. A unique feature of this system, which clearly distinguishes it from the previous designs, is its capacity for passive targeted energy transfer from the LO to the NES even at low energies. This is in contrast to the previous NES designs based on purely cubic stiffness nonlinearities, where there exists a energy threshold below which no targeted energy transfer was possible. Indeed, when the LO is excited by a low-energy impulse, two main nonlinear passive energy transfer mechanisms from the LO to the NES arise: aperiodic (chaotic) cross-well oscillations and in-well nonlinear beats. The description of each of these nonlinear mechanisms is facilitated by reducing the full system to an equivalent single degree of freedom reduced-order forced oscillator; this dimension reduction relies on the analogy between phase and configuration spaces. Melnikov analysis and Lyapunov characteristic exponents are then adopted to identify the region of existence of chaotic cross-well oscillations which enable efficient targeted energy transfer to the NES even at low-intensity applied impulses to the LO. Asymmetric limiting phase trajectories are investigated to derive analytical approximations of the in-well nonlinear beats, which provide the second mechanism for energy transfer. The proposed approaches describe and predict the beneficial dynamic phenomena governing the targeted energy transfer from the LO to the NES, thus providing valuable tools to design efficient passive energy absorption devices for low amplitude ambient vibrations.

\section{ACKNOWLEDGMENTS}

The authors are grateful to M.A. Kovaleva for fruitful discussions and computational help.

\section{APPENDIX: REDUCED SYSTEM COMPLEXIFICATION}

In order to describe the asymmetric LPTs, the reduced order system (23) is expressed in complex form. By denoting $\dot{u}=v$, Eq. (23) becomes

$$
\dot{v}+\mu \varepsilon^{2} v+u+4 \alpha \varepsilon u^{2}+8 \beta \varepsilon^{2} u^{3}=2 \varepsilon^{2} F \sin \left(\frac{\omega}{\omega_{0}} \tau\right) .
$$

Then, by applying complexification, the variables $\psi=v+j u$ and $\psi^{*}=v-j u$ are introduced and (A1) becomes

$$
\begin{aligned}
\frac{d \psi}{d \tau_{0}} & -j \psi+\varepsilon^{2} \mu\left(\psi-\psi^{*}\right)-\alpha \varepsilon\left(\psi-\psi^{*}\right)^{2}+j \beta \varepsilon^{2}\left(\psi-\psi^{*}\right)^{3} \\
& =2 \varepsilon^{2} F \sin \left(\frac{\omega}{\omega_{0}} \tau_{0}\right),
\end{aligned}
$$


with initial condition $\psi=0$. At this point, the condition of $1: 1$ resonance is imposed by setting $\omega=\omega_{0}$. By setting $\psi=\varphi e^{j \tau_{0}}, \psi^{*}=\varphi^{*} e^{-j \tau_{0}}$, the complex slow modulation $\varphi$ is governed by

$$
\begin{aligned}
\frac{d \varphi}{d \tau_{0}} & +\varepsilon^{2} \mu\left(\varphi+\varphi^{*} e^{-2 j \tau_{0}}\right) \\
& -\alpha \varepsilon\left(\varphi^{2} e^{j \tau_{0}}+\varphi^{*^{2}} e^{-3 j \tau_{0}}-2|\varphi|^{2} e^{j \tau_{0}}\right) \\
& +\varepsilon^{2} j \beta\left(\varphi^{3} e^{2 j \tau_{0}}-\varphi^{*^{3}} e^{-4 j \tau_{0}}-3|\varphi|^{2} \varphi+3|\varphi|^{2} \varphi^{*} e^{-2 j \tau_{0}}\right) \\
= & 2 \varepsilon^{2} e^{-j \tau_{0}} F \sin \left(\frac{\omega}{\omega_{0}} \tau_{0}\right) .
\end{aligned}
$$

After performing averaging of this complex differential equation with respect to the "fast" normalized frequency equal to unity, we apply the multiple scale method by considering the "fast" time scale $\tau_{0}$ and the "slow" time scales $\tau_{1}=\varepsilon \tau_{0}, \tau_{2}=\varepsilon^{2} \tau_{0}, \ldots$. Following that, the slow modulation $\varphi$ is expanded according to $\varphi=\varphi_{0}+\varepsilon \varphi_{1}+\varepsilon^{2} \varphi_{2}+\cdots$.

Substituting the previous expressions to the averaged equation and considering only the leading order approximation, the equation governing the main asymptotic approximation is given by

$$
\frac{d \varphi_{0}}{d \tau_{2}}+\mu \varphi_{0}-\alpha^{*} j\left|\varphi_{0}\right|^{2} \varphi_{0}=-j F e^{j s \tau_{2}},
$$

where $\alpha^{*}=3 \beta-\frac{20}{3} \alpha^{2}$ and $\varphi_{0}(0)=0$; by setting $\varphi_{0}=a e^{j \delta}$, and considering separately the real and imaginary parts of Eq. (A4), we obtain Eqs. (25).

${ }^{1}$ L. I. Manevitch, G. Sigalov, F. Romeo, L. A. Bergman, and A. F. Vakakis, "Dynamics of a linear oscillator coupled to a bistable light attachment: Analytical study,” J. Appl. Mech. 81, 041011 (2013).

${ }^{2}$ F. Romeo, G. Sigalov, L. A. Bergman, and A. F. Vakakis, "Dynamics of a linear oscillator coupled to a bistable light attachment: Numerical study," J. Comput. Nonlinear. Dyn. 10, 011007 (2014).

${ }^{3}$ A. F. Vakakis, O. Gendelman, L. A. Bergman, D. M. McFarland, G. Kerschen, and Y. S. Lee, Passive Nonlinear Targeted Energy Transfer in Mechanical and Structural Systems: I and II (Springer Verlag, 2008).

${ }^{4}$ M. I. Dykman, S. M. Soskin, and M. A., Krivoglaz, "Spectral distribution of a nonlinear oscillator performing Brownian motion in a double-well potential," Physica A 133, 53-73 (1985).

${ }^{5}$ L. N. Virgin, R. H. Plaut, and C.-C. Cheng, "Prediction of escape from a potential well under harmonic excitation," Int. J. Non-Linear Mech. 27(3), 357-365 (1992).
${ }^{6}$ B. P. Mann and B. A. Owens, "Investigations of a nonlinear energy harvester with a bistable potential well," J. Sound Vib. 329(9), 1215-1226 (2010).

${ }^{7}$ M. F. Daqaq, "Transduction of a bistable inductive generator driven by white and exponentially correlated Gaussian noise," J. Sound Vib. 330, 2554-2564 (2011).

${ }^{8}$ S. C. Stanton, B. P. Mann, and B. A. M. Owens, "Melnikov theoretic methods for characterizing the dynamics of the bistable piezoelectric inertial generator in complex spectral environments," Physica D 241(6), 711-720 (2012).

${ }^{9}$ D. J. Tweten and B. P. Mann, "Experimental investigation of colored noise in stochastic resonance of a bistable beam," Physica D 268, 25-33 (2014).

${ }^{10} \mathrm{Q}$. He and M. F. Daqaq, "New insights into utilizing bistability for energy harvesting under white noise," J. Vib. Acoust. 137(2), 021009 (2015).

${ }^{11}$ T. Mizuno, T. Toumiya, and M. Takasaki, "Vibration isolation using negative stiffness,” JSME Int. J., Ser. C 46(3), 807-821 (2003).

${ }^{12}$ T. Jaglinski and R. S. Lakes, "Anelastic instability in composites with negative stiffness inclusions," Philos. Mag. Lett. 84(12), 803-810 (2004).

${ }^{13}$ C. M. Lee, V. N. Goverdovskiy, and A. I. Temnikov, "Design of springs with 'negative' stiffness to improve vehicle driver vibration isolation," J. Sound Vib. 302, 865-874 (2007).

${ }^{14}$ A. Carrella, "Passive vibration isolators with high-static-low-dynamicstiffness," Ph.D. thesis (Institute of Sound and Vibration Research, University of Southampton, 2008).

${ }^{15}$ L. Dong and R. S. Lakes, "Advanced damper with negative structural stiffness elements," Smart Mater. Struct. 21, 075026 (2012).

${ }^{16}$ X. Huang, X. Liu, J. Sun, Z. Zhang, and H. Hua, "Vibration isolation characteristics of a nonlinear isolator using Euler buckled beam as negative stiffness corrector: A theoretical and experimental study," J. Sound Vib. 333, 1132-1148 (2014).

${ }^{17}$ F. Liu, S. Theodossiades, D. M. McFarland, L. A. Bergman, and A. F. Vakakis, "Tailoring strongly nonlinear negative stiffness," J. Mech. Des. 136(2), 024501 (2013).

${ }^{18}$ J. Guckenheimer and P. Holmes, Nonlinear Oscillations, Dynamical Systems, and Bifurcations of Vector Fields (Springer-Verlag, New York, 1985).

${ }^{19}$ S. Wiggins, Introduction to Applied Nonlinear Dynamical Systems and Chaos (Springer-Verlag, New York, 1990).

${ }^{20}$ A. J. Lichtenberg and M. A. Lieberman, Regular and Chaotic Dynamics (Springer-Verlag, New York, 1992).

${ }^{21}$ G. Benettin, L. Galgani, A. Giorgilli, and J. M. Strelcyn, "Lyapunov characteristic exponents for smooth dynamical systems and for Hamiltonian systems; A method for computing all of them. Part 2: Numerical application," Meccanica 15, 21-30 (1980).

${ }^{22}$ L. I. Manevitch, "New approach to beating phenomenon in coupled nonlinear oscillatory chains," Arch. Appl. Mech. 77(5), 301-312 (2007).

${ }^{23}$ L. I. Manevitch and V. V. Smirnov, "Limiting phase trajectories and the origin of energy localization in nonlinear oscillatory chains," Phys. Rev. E 82, 036602(1-9) (2010).

${ }^{24}$ A. F. Vakakis, L. I. Manevitch, Yu. V. Mikhlin, V. N. Pilipchuk, and A. A. Zevin, Normal Modes and Localization in Nonlinear Systems (Wiley Interscience, New York, 1996).

${ }^{25}$ E. L. Manevitch and L. I. Manevitch, "Limiting phase trajectories (LPT) in a 1 dof asymmetric system with damping and 1:1 resonance," in Proceedings of the 10th Conference on Dynamical Systems Theory and Applications, Lodz, Poland, 7-10 December 2009. 